\title{
Theoretical prediction of morphological selection in amphiphilic systems
}

\author{
Karl Glasner* \\ Department of Mathematics, University of Arizona, Tucson, Arizona 85721, USA
}

(Received 12 July 2019; published 11 December 2019)

\begin{abstract}
Biological and synthetic amphiphilic systems exhibit a wide range of morphologies. A density functional model for amphiphilic polymer phase mixtures is utilized to quantify localized equilibria and their stability, and ultimately predict and explain morphological preference. This is done by utilizing matched asymptotic expansions, which produces explicit connections between model parameters and macroscopic properties of equilibrium structures. Bilayers, cylindrical, and spherical micelle and vesicle configurations are found, and formulas which connect their geometry to ambient chemical potential are derived. Dynamics are studied in the context of a free boundary problem which describes the evolution of the hydrophobic-solvent domain interface. Linearization of this problem is used to explicitly determine growth rates and parameter regions of stability. All equilibria are found to have two branches of solutions terminating at a fold in the bifurcation diagram which signals the crossover from competitive stability to instability leading to ripening behavior. Ideally flat bilayers are determined to always possess a long wavelength buckling instability, suggesting that curved structures should be generically preferred. Spherical micelles exhibit morphological instabilities which are suppressed by large enough surface tension. Cylindrical micelles may have short-wavelength pearling and long-wavelength Rayleigh-Plateau-type instabilities. In addition, ideally infinite cylinders have an undulatory instability, suggesting that only finite length structures should be observed. A morphological phase diagram can be assembled which takes into account both existence and stability of different geometries. Consistent with experimental evidence, a bifurcation sequence from spheres to cylinders to vesicles is found as either surface tension or polymer composition increases. Coexistence of different stable morphologies is also observed.
\end{abstract}

DOI: 10.1103/PhysRevE.100.062501

\section{INTRODUCTION}

Amphiphilic molecules are ubiquitous in cellular biology, where they form membranes and other intracellular structures. Synthetic amphiphiles, commonly made from block copolymers, are becoming increasingly important in the engineering of self-assembled materials [1-5]. In both the biological and polymer contexts, amphiphiles are characterized by their mixed affinity with a solvent. The hydrophilic part of an amphiphile has an energetic preference (quantified by a negative Flory-Huggins parameter [6]) to mix with the solvent, whereas the hydrophobic part has the opposite affinity. The resulting compromise between these two effects is the creation of a wide array of nanoscale structures.

Experiments with synthetic amphiphiles reveal a variety of possible morphologies [7-9], including cylindrical ("rods") and spherical micelles, and vesicles comprising bilayer sheets. In addition, the presence of defects such as end caps and junctions promotes formation of complex networks. Preference toward different geometries is influenced by several factors, including the strength of intermolecular interactions, polymer composition, and polymer or solvent volume fraction $[7,8]$.

A wide variety of mesoscale models have been used to explore amphiphilic systems. This includes coarse-grained approaches [10-12] and phenomenological continuum equa-

*kglasner@math.arizona.edu tions $[13,14]$. While capturing some of the desired mechanical properties of amphiphilic bilayers and pore configurations, it is not straightforward in these approaches to connect model parameters to the underlying chemical details.

A widely adopted framework which accounts for molecular characteristics of polymer systems is self-consistent field theory (SCFT) [15-17]. This was originally developed to predict heterogeneous equilibrium configurations utilizing the Gaussian random walk model for polymer chains [18], but has also been formulated for time-dependent systems as dynamic SCFT [19] and external potential dynamics [20]. While it has been used successfully in simulation of complex micelles [21,22], dynamic versions (especially in three dimensions) involve huge numerical costs. In addition, there is remarkably little understanding of these models from an analytical point of view, undoubtedly due to the complexity of the nonlocal mapping from density to field variables. This fact places strong limitations on both numerical and analytical study and limits a full accounting of the model's predictions over a wide range of parameters.

A complementary approach to SCFT is density functional theory, which has a common root [23] but makes approximations of nonlocal interactions [24-26]. Like SCFT, it was originally formulated for investigating equilibria, but also readily extends to dynamic circumstances [27]. Both paradigms have successfully reproduced various aspects of polymer systems [28], including phase diagrams for diblock mixtures [26,29], nanoparticle formation in copolymersolvent mixtures [30,31], and amphiphilic structure 

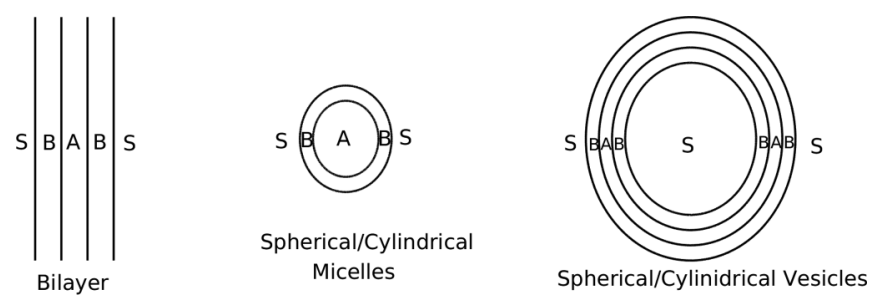

FIG. 1. Schematic representation of the families of amphiphilic structures considered in this paper. The $A, B, S$ regions are where there is a preponderance of hydrophobic, hydrophilic, and solvent species, respectively.

formation [32,33]. These models are derivatives of phase field models such as the Cahn-Hilliard theory of phase separation [34], which have proven to yield considerable mathematical understanding, including good quantitative approximations. This tractability motivates the present work, wherein analytical expressions are derived that make explicit connections between physical parameters and predictions of morphology.

It is reasonable to inquire if continuum models may be used for nearly molecular sized structures. Typical core widths of amphiphilic bilayers range from 3 to $5 \mathrm{~nm}$ for biological lipids to 8 to $21 \mathrm{~nm}$ in experiments with block copolymers [7]. Although the molecular size (measured, for example, by the unperturbed radius of gyration) is only slightly smaller, it should be noted that SCFT (and by extension DFT) describe composition variations not at the molecular level, but at the considerably smaller monomer scale. Evidence of the applicability of continuum modeling of block copolymer architectures has a very long history [17].

This paper studies various families of symmetric equilibria in a generalized density functional model of amphiphilic mixtures (Fig. 1). Localized equilibria are characterized as fixed points of an evolution equation, which may alternately be viewed as critical points of a suitably constrained free energy. It should be emphasized that these do not represent a global energy minimum (which in the present model would be a homogeneous mixture of polymer and solvent). In addition, since the configurations under consideration do not represent homogeneous mixtures, basic notions of thermodynamic stability do not apply. The realization of localized structures therefore depends both on the way the system is prepared as well as whether critical points represent local energy minimizers (i.e., metastable states). This paper is focused on the latter condition and is studied by considering the effect of small perturbations.

It is useful to subdivide the roles played by perturbations induced by neighboring structures and those which arise as localized, mass-preserving fluctuations. We will refer to the former as competitive stability and the latter as morphological stability. Competition of localized equilibria in diffusiondriven systems arises from mass exchange resulting from imbalances of the local chemical potential(s). In classical Ostwald ripening $[35,36]$, this leads to larger particles growing at the expense of smaller ones, a situation of competitive instability. In contrast, localized domains in polymer systems may or may not coexist with their neighbors [31]. One way to characterize competitive stability is through the relationship between a structure's equilibrium chemical potential (given here by $v_{\infty}$ ) and its volume $V$. If $d v_{\infty} / d V>0$ among a family (or families) of equilibria, then mass exchange will be from larger domains to smaller ones, a situation of competitive stability. On the other hand, morphological instabilities may result in widespread changes in domain geometry. Here we explicitly quantify both mechanisms of instability and explore the consequences for morphological preference.

Section II introduces the model framework which we utilize to study the evolution of amphiphilic mixtures. The analytic construction of symmetric equilibria using the method of matched asymptotic expansions is summarized in Sec. III. Linearized stability is computed in Sec. IV, which gives predictions of both competitive and morphological instabilities. Finally, the theoretical predictions of the analysis are discussed in the context of the morphological phase diagram (Sec. V). Details of the asymptotic expansion calculations are deliberately deferred to Appendices A and B.

\section{DENSITY FUNCTIONAL MODEL}

A variety of formulations of density functional models for heterogeneous polymer systems exist. The formulation used here is a simplification of three-phase models $[26,37]$ which have been employed for mixtures of solvent and diblock copolymers. Volume fractions of the hydrophobic phase $A$ and hydrophilic phase $B$ are given by $\phi_{A}, \phi_{B}$, respectively, so that under a typical incompressibility assumption the solvent volume fraction is $1-\phi_{A}-\phi_{B}$. A convenient choice of order parameters is [37]

$$
\Phi=(1-f) \phi_{A}-f \phi_{B}, \quad \Psi=f \phi_{A}+(1-f) \phi_{B},
$$

where $f$ is volume fraction of the hydrophobic phase.

The simplest form for free energy (in units of the Boltzmann energy $k T$ ) of the system under consideration may be written $[37,38]$

$$
\begin{aligned}
F= & \int_{\mathbb{R}^{d}} W(\Phi, \Psi)+G_{11}|\nabla \Phi|^{2}+G_{12}|\nabla \Phi \cdot \nabla \Psi|^{2} \\
& +G_{22}|\nabla \Psi|^{2} d \boldsymbol{x}+\frac{\alpha}{2} \int_{\mathbb{R}^{d}} \int_{\mathbb{R}^{d}} K\left(\boldsymbol{x}, \boldsymbol{x}^{\prime}\right) \Phi(\boldsymbol{x}) \Phi\left(\boldsymbol{x}^{\prime}\right) d \boldsymbol{x} d \boldsymbol{x}^{\prime} .
\end{aligned}
$$

The scaled bulk free energy $W$ is specified below and can alternately be written as a function of $\left(\phi_{A}, \phi_{B}\right)$ by virtue of (1). The coefficients $G_{i j}$ and $\alpha$ may be derived from molecular considerations using the random phase approximation $[23,28,39]$. For simplicity, we take $G_{11}=G=G_{22}$ and $G_{12}=0$. Note this choice affects only the phase interface structure, which has no consequence in the present context other than to determine the surface energy parameter [see, e.g., (13)]. The last term in (2) penalizes separation of hydrophobic and hydrophilic components, which would be expected of molecularly bonded phases. The nonlocal interaction kernel $K()$ is taken to be the Laplacian Green's function. 


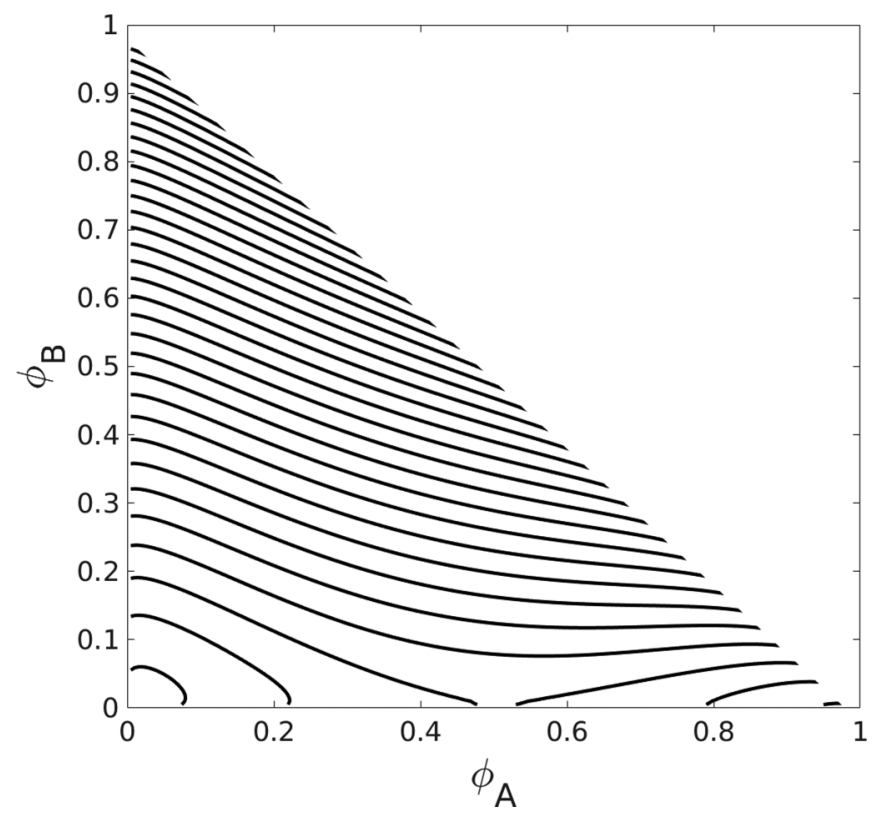

FIG. 2. Contours of modified potential with $\chi_{A B}=4=\chi_{A S}$, $\chi_{B S}=-1, \mu_{A}=0$, and $\mu_{B}=5$. The minima are roughly at $\left(\phi_{A}, \phi_{B}\right)=(0,0)$ and $(1,0)$, corresponding to pure solvent and hydrophobic phases, respectively.

A completely dimensionless formulation can be obtained by scaling lengths by $\alpha^{-1 / 5}$, which results in

$$
\begin{aligned}
F= & \int_{\mathbb{R}^{d}} W(\Phi, \Psi)+\frac{\epsilon^{2}}{2}|\nabla \Phi|^{2}+\frac{\epsilon^{2}}{2}|\nabla \Psi|^{2} d \boldsymbol{x} \\
& +\frac{1}{2} \int_{\mathbb{R}^{d}} \int_{\mathbb{R}^{d}} K\left(\boldsymbol{x}, \boldsymbol{x}^{\prime}\right) \Phi(\boldsymbol{x}) \Phi\left(\boldsymbol{x}^{\prime}\right) d \boldsymbol{x} d \boldsymbol{x}^{\prime} .
\end{aligned}
$$

Here the dimensionless interface width $\epsilon=\sqrt{2} G^{1 / 2} \alpha^{-1 / 10}$, which will be assumed small.

A common expression for the bulk free energy $W\left(\phi_{A}, \phi_{B}\right)$ is given by [26]

$$
W=\sum_{p=A, B, S} C_{p} \phi_{p} \ln \phi_{p}+\sum_{p q=A B, B S, A S} \frac{\chi_{p q}}{2} \phi_{p} \phi_{q} .
$$

The amphiphilic nature of the system requires $\chi_{A S}>0$ and $\chi_{B S}<0$. In addition, if the polymer is in the phase-separated regime, then $\chi_{A B}>0$. Since the underlying variational problem has a constraint which conserves volume of the phases, the potential may be altered by addition of any linear function $W \rightarrow W+\mu_{A} \phi_{A}+\mu_{B} \phi_{B}$, which is equivalent to setting a gauge for the chemical potentials. For typical values of interaction parameters, the modified potential can be arranged so that it has minima close to $\left(\phi_{A}, \phi_{B}\right)=(0,0)$ and $(1,0)$, corresponding to solvent and hydrophobic phases (Fig. 2). In addition, the minimum of $W\left(\phi_{A}, \cdot\right)$ for fixed $\phi_{A}$ is roughly where $\phi_{B} \sim 0$. In light of this observation, rather than specializing to (4), this paper considers a broad class of potentials $W\left(\phi_{A}, \phi_{B}\right)$ satisfying the following conditions:

(1) There are two minima at $(0,0)$ and $(1,0)$, representing pure solvent and pure hydrophobic phase

(2) For sufficiently small $\phi_{B}$, the potential is convex in $\phi_{B}$.
Notice that the composition parameter $f$ is still retained in the model via (1). For purposes of illustration and numerical experiments, we will take $f=1 / 2$ and use a convenient choice for the potential:

$$
W\left(\phi_{A}, \phi_{B}\right)=\chi_{A S} \phi_{A}^{2}\left(1-\phi_{A}\right)^{2}+\phi_{B}^{2} .
$$

We emphasize, however, the analytical derivations which follow are not specialized to this choice, and the potential must satisfy only the modest requirements listed above. In addition, the analysis provides a way to map physical parameters such as those in (4) to the surface energy energy [see, e.g., (13) and the related discussion].

Generalized chemical potential fields $\nu, \mu$ may be defined in terms of variations of the free energy $\mu=\delta F / \delta \Phi, v=$ $\delta F / \delta \Psi$, and these drive diffusive dynamics via

$$
\left(\begin{array}{l}
\Phi_{t} \\
\Psi_{t}
\end{array}\right)=\nabla \cdot\left[\boldsymbol{M \nabla}\left(\begin{array}{c}
\delta F / \delta \Phi \\
\delta F / \delta \Psi
\end{array}\right)\right] .
$$

The mobility tensor $\boldsymbol{M}$ describes the rates of diffusion and cross-diffusion and for simplicity of presentation will be taken to be the identity.

\section{SYMMETRIC EQUILIBRIA}

Amphiphilic structures which are at thermodynamic equilibrium with their surroundings may be studied by regarding them as constrained equilibria of (3). The corresponding Euler-Lagrange system is

$$
\begin{gathered}
\Delta\left(-\epsilon^{2} \Delta \Phi+W_{\Phi}\right)=\Phi \\
-\epsilon^{2} \Delta \Psi+W_{\Psi}=v_{\infty} \equiv W_{\Psi}\left(0, \Psi_{\infty}\right) .
\end{gathered}
$$

The Lagrange multiplier $v_{\infty}$ can be associated with a constraint that conserves polymer volume; alternatively, it can be viewed as the ambient chemical potential for the polymer species. In general, for each class under consideration there is a family of equilibria parameterized by size (width, radius, etc.) which determines the value of $v_{\infty}$; it is this relationship that will be calculated explicitly below. Finally, it is supposed that the mixture is spatially homogeneous far away from the structure, leading to the conditions

$$
\lim _{r \rightarrow \infty} \Phi=0, \quad \lim _{r \rightarrow \infty} \Psi=\Psi_{\infty} .
$$

While the structures of interest are all three-dimensional, symmetry considerations allow for the study of equilibria in lower dimension $d$. For ideally flat bilayer sheets, $d=1$; for cylindrical micelles and vesicles, $d=2$; spherical micelles and vesicles have $d=3$. In each case the Laplacian is restricted to radially symmetric fields,

$$
\Delta u=\Delta_{r} u \equiv \frac{1}{r^{d-1}}\left(r^{d-1} u_{r}\right)_{r} .
$$

Solutions of (7) and (8) may be found by exploiting the smallness of $\epsilon$ by using multiscale expansions for internal layers (e.g., Refs. [27,40]). This section summarizes the principal results of these computations, which are described in complete detail in Sec. III A. A mathematically rigorous study of this problem will be reported elsewhere [41]. 


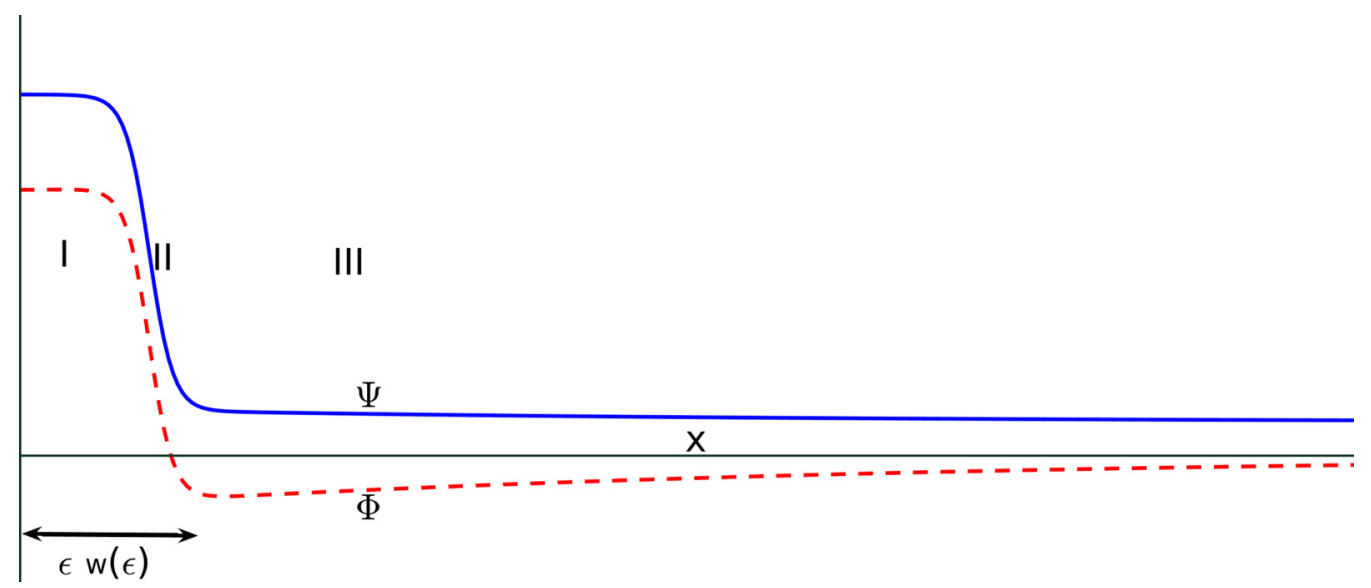

FIG. 3. Regions for matched asymptotic expansion of symmetric bilayer-type equilibrium. Region I is the hydrophobic core, region II is the interface, and region III contains the hydrophilic tail. Micelle-type equilibria use the same setup, with $x$ replaced by the radial variable $r$ and core width $\epsilon w$ replaced by core radius $R$. The amplitudes of the $\Phi$ and $\Psi$ profiles are roughly one.

\section{A. Bilayer equilibria}

Solutions to (7) and (8) are constructed using expansions over three regions (see Fig. 3). The hydrophobic phase region I has width $\epsilon w\left(v_{\infty}\right)$, where it is supposed that $w \gg 1$, and to leading order $(\Phi, \Psi) \approx(1-f, f)$, corresponding to phase $A$. Region III comprises both the solvent and the hydrophilic phases, whose density decays as $\exp (-x / \sqrt{\beta})$. In the dimensionless formulation, $\sqrt{\beta}$ measures the hydrophilic domain width and depends only on the bulk potential by

$$
\beta \equiv \operatorname{det} H^{0} / H_{\Psi \Psi}^{0}, H^{0} \equiv \nabla^{2} W(0,0)=\left(\begin{array}{ll}
H_{\Phi \Phi}^{0} & H_{\Phi \Psi}^{0} \\
H_{\Phi \Psi}^{0} & H_{\Psi \Psi}^{0}
\end{array}\right)
$$

The two phase regions are separated by a diffuse interface (region II), whose role here is to merely connect the behavior of the expansion on either side.

Matching of the chemical potentials $v, \mu$ across all three regions leads to a relationship between the bilayer width and

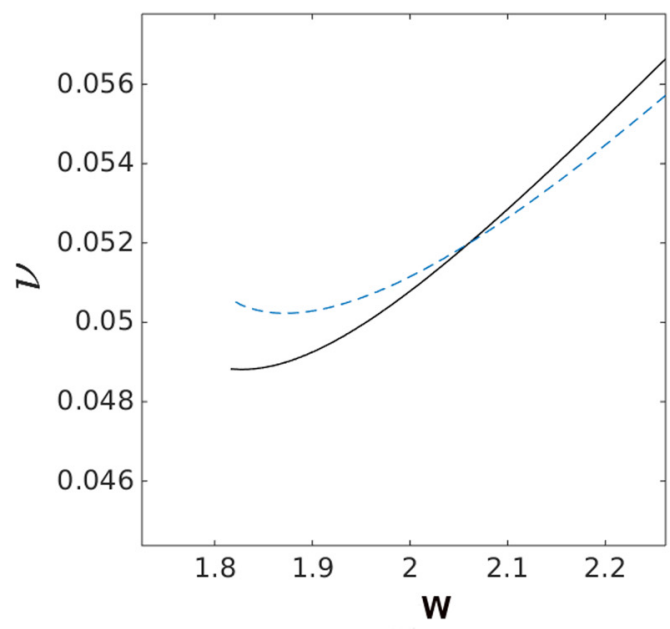

chemical potential

$$
\begin{aligned}
v_{\infty} & =\frac{1}{\gamma}\left[(1-f) \sqrt{\beta} \epsilon w+\frac{4 \lambda^{2}}{1-f} \exp (-2 \lambda w)\right], \\
\gamma & \equiv \frac{f}{1-f}+\frac{H_{\Phi \Psi}^{0}}{H_{\Psi \Psi}^{0}} .
\end{aligned}
$$

Here $\lambda$ is the eigenvalue of $H^{0}$, taken to be of multiplicity two for simplicity.

The analytical result (11) was compared to numerical computations of solutions of (7) and (8). The latter were found by evolving the dynamic equations toward a steady state, using a periodic domain much larger than the bilayer width. Other details of the algorithm can be found elsewhere [42]. The numerical comparison uses the specific potential (5) with $\chi_{A S}=1$, from which one finds that $\beta=4$ and eigenvalues of $H^{0}$ are both equal to $\lambda=2$.

Figure 4 shows the comparison between analytical and numerical results for $\epsilon=2.5 \times 10^{-2}$ and $\epsilon=6.25 \times 10^{-3}$. Significantly, both the analysis and numerics show the existence of a minimum value for $v_{\infty}$ within the family of equilibria.

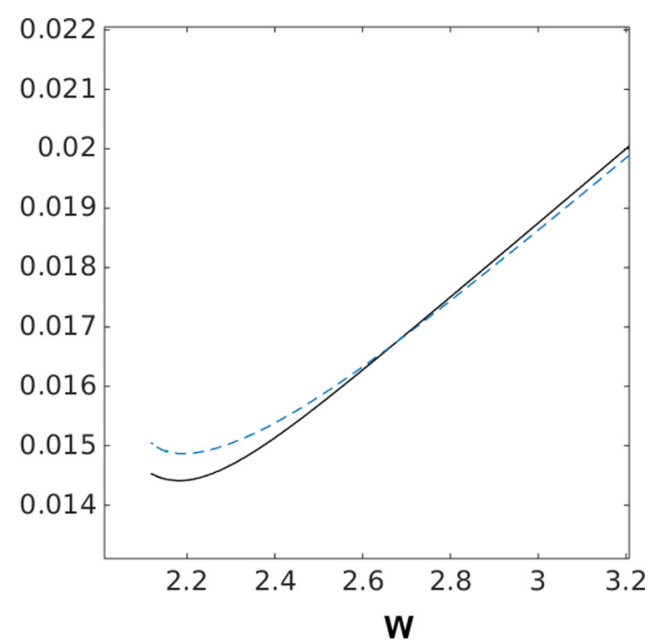

FIG. 4. Comparisons of the analytical prediction (11) (solid/black) and numerical computations (dotted/blue) of the family of bilayer-type solutions. (a) $\epsilon=2.5 \times 10^{-2}$; (b) $\epsilon=6.25 \times 10^{-3}$. 

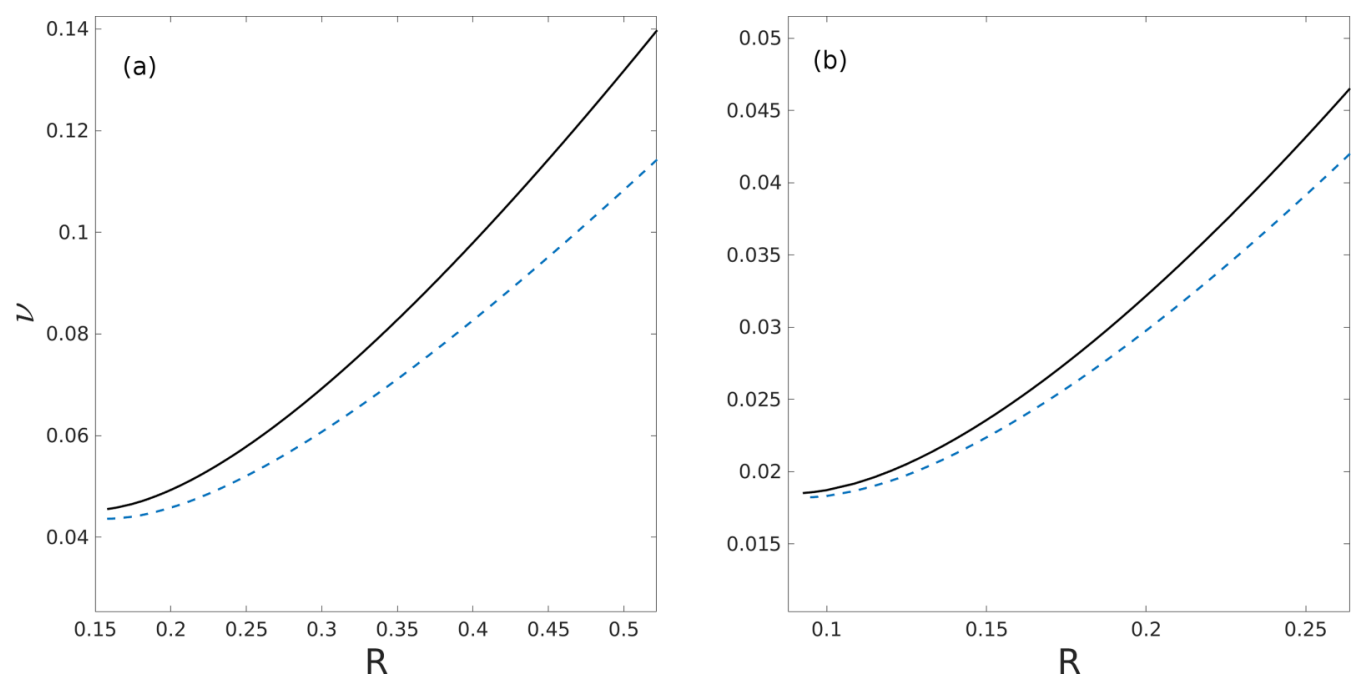

FIG. 5. Comparisons of the analytical prediction (12) [solid (black)] and numerical computations [dotted (blue)] of radial equilibria for dimension $d=2$. (a) $\epsilon=1.25 \times 10^{-2}$; (b) $\epsilon=3.125 \times 10^{-3}$.

For large bilayer widths, the $v_{\infty}(w)$ relation becomes linear in both cases, whereas when the width becomes small enough, the bilayer structures become competitively unstable and collapses. Unsurprisingly, the predictions of the analysis cease to agree with numerical findings when either $w \gg \ln \epsilon$ or $w=$ $O(1)$. The former case includes the unphysical circumstance of bilayers much larger than the constituent molecules. The latter case is simply reflects a breakdown of the sharp interface (strong segregation) assumption underlying the analysis.

\section{B. Micelle equilibria}

Radially symmetric solutions of (7) and (8) may be approximated using an expansion scheme similar to the bilayer case. The interface region is assumed to exist at radius $r \approx R$, where the core and solvent or hydrophilic domains are given by $r<R$ and $r>R$, respectively. The results of the asymptotic calculations (Sec. III A 2) again yield relationships between

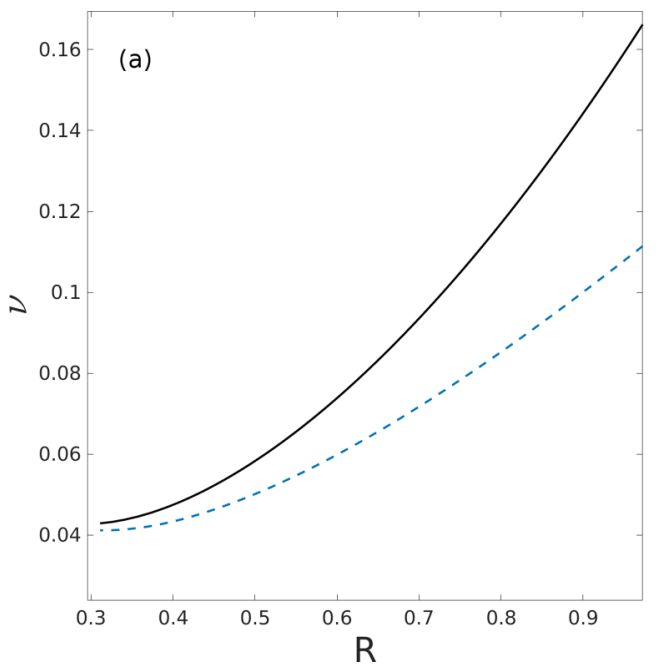

structure size and chemical potential:

$$
\nu_{\infty}=\frac{1}{\gamma}\left\{\begin{array}{ll}
\frac{\epsilon \sigma}{(1-f) R(\epsilon)}-\frac{R(\epsilon)^{2}}{2} \ln [R(\epsilon) / 2 \sqrt{\beta}], & d=2 \\
\frac{2 \epsilon \sigma}{(1-f) R(\epsilon)}+\frac{R(\epsilon)^{2}}{3}, & d=3
\end{array} .\right.
$$

Here $\sigma$ represents dimensionless surface energy density and arises as in other phase field problems from the interface profile $\left(\Phi_{h}, \Psi_{h}\right)(\zeta)$ as

$$
\sigma=\int_{-\infty}^{\infty}\left(\Phi_{h}^{\prime}\right)^{2}+\left(\Psi_{h}^{\prime}\right)^{2} d \zeta
$$

Computations of radially symmetric micelle-type equilibria were compared to the approximations (12). Using (5) with $\chi_{A S}=1$, it is found that $\Phi_{h}=\Psi_{h}=(\tanh \zeta+1) / 4$ and $\sigma=$ $1 / 6$. Figures 5 and 6 compare the analytical and numerical results for $\epsilon=1.25 \times 10^{-2}$ and $\epsilon=3.125 \times 10^{-3}$. The predictions of the analysis appear to be in close agreement with

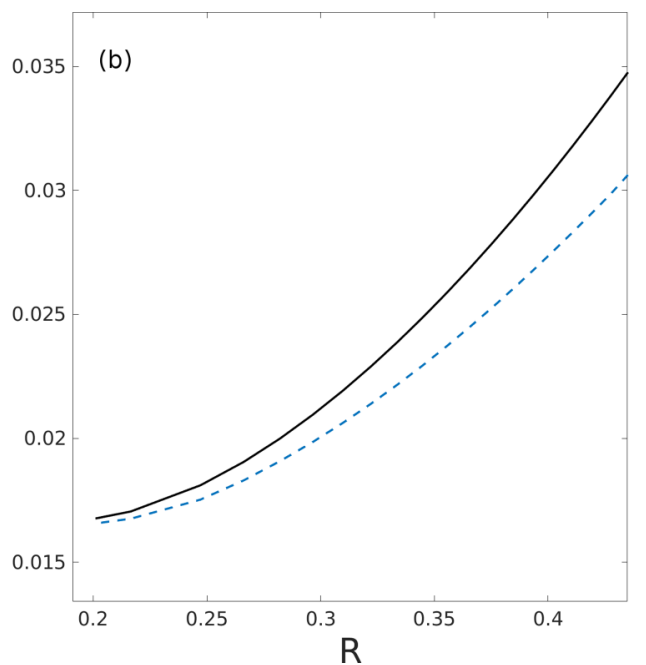

FIG. 6. Comparisons of the analytical prediction (12) [solid (black)] and numerical computations [dotted (blue)] of radial equilibria for dimension $d=3$. (a) $\epsilon=1.25 \times 10^{-2}$; (b) $\epsilon=3.125 \times 10^{-3}$. 


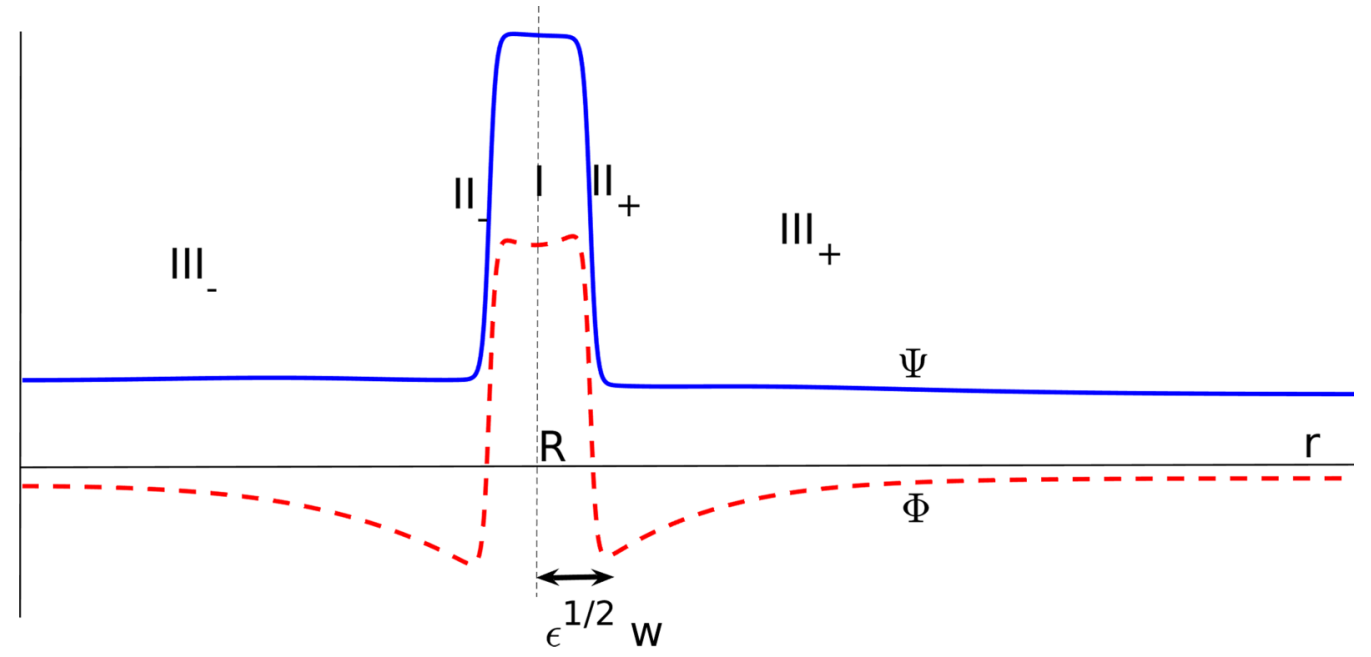

FIG. 7. Regions used in asymptotic expansion of vesicle-type solutions.

numerical findings but of course become worse as $R$ becomes large where the scaling assumptions are not respected.

As in the case of bilayers, there is a critical size $R^{*}$ corresponding to the minimum of $v_{\infty}(R)$. For $R>R^{*}, v_{\infty}^{\prime}(R)>0$, which implies competitive stability, as will be discussed in Sec. IV. In contrast, the branch for $R<R^{*}$ (not shown in Figs. 5 and 6) is competitively unstable. In the absence of other instabilities or competition from other morphologies, a collection of micelles is in general driven to a radius close to the threshold $R^{*}$ [31].

\section{Vesicle equilibria}

For radially symmetric vesicle domains, there are two length scales, the bilayer width and the radius. Consistency of the matched asymptotic calculation requires that the width and $v_{\infty}$ have the same scaling in $\epsilon$ and that the width scales as $\epsilon^{1 / 2}$. To this end, the hydrophilic or solvent domain regions are assumed to exist where $r>R+\epsilon^{1 / 2} w$ and $r<R-\epsilon^{1 / 2} w$, where $R$ is the effective radius. These regions are separated by a curved bilayer, whose half-width is $\epsilon^{1 / 2} w$ (Fig. 7). The calculation detailed in Sec. III A 3 determines relationships between both $w$ and $R$ and the equilibrium chemical potential:

$$
v_{\infty}=\sqrt{\frac{\sigma(d-1)}{(1-f) R \bar{A}(R) \bar{w}(R)}},
$$

where

$$
\bar{w}(R)=\frac{\gamma}{2(1-f) \sqrt{\beta}} \begin{cases}I_{0}^{\prime}(R / \sqrt{\beta}) / I_{0}(R / \sqrt{\beta})-K_{0}^{\prime}(R / \sqrt{\beta}) / K_{0}(R / \sqrt{\beta}), & d=2 \\ 1+\operatorname{coth}(R / \sqrt{\beta}), & d=3\end{cases}
$$

and

$$
\bar{A}(R)=-\frac{\gamma}{2 \sqrt{\beta}}\left\{\begin{array}{ll}
I_{0}^{\prime}(R / \sqrt{\beta}) / I_{0}(R / \sqrt{\beta})+K_{0}^{\prime}(R / \sqrt{\beta}) / K_{0}(R / \sqrt{\beta}), & d=2 \\
-1-2 \sqrt{\beta} / R+\operatorname{coth}(R / \sqrt{\beta}), & d=3
\end{array} .\right.
$$

Comparisons of numerical and analytic expressions are shown in Fig. 8, using the same potential and parameters as the previous cases. Convergence of the numerical solutions to the analytic approximations are seen as the small parameter $\epsilon$ is decreased.

The dependence of vesicle width on radius is reported in Fig. 9 (left). In the case of both large cylindrical and spherical vesicles, the width approaches a constant. This fact is consistent with experiments with synthetic amphiphiles [7] and some theories of membrane energy [43]. Curiously, however, cylinders exhibit a nonmonotonic dependence in width at smaller radii.

Competitive stability can be ascertained from the dependence of $v_{\infty}$ on core size, defined as $4 \pi R w$ for cylinders and
$8 \pi w R^{2}$ for spheres. Figure 9 (right) shows these relationships for both cylinders and spheres. Cylindrical vesicles follow a pattern similar to the micelle cases, wherein competitive stability is seen only for structures larger than a certain size. This indicates that narrow cylinders should be out-competed by larger neighboring structures.

The results for spherical vesicles show some marked differences from the configurations previously considered. Solutions cease to exist for small enough radius, which might be expected if the inner radius is comparable to the bilayer width. More surprisingly, competitive instability is encountered for radii which are greater than a critical value (here $R^{*} \approx 2.5$ ). This appears to suggest that large vesicles would be subject to Ostwald ripening behavior. On the other hand, a more 

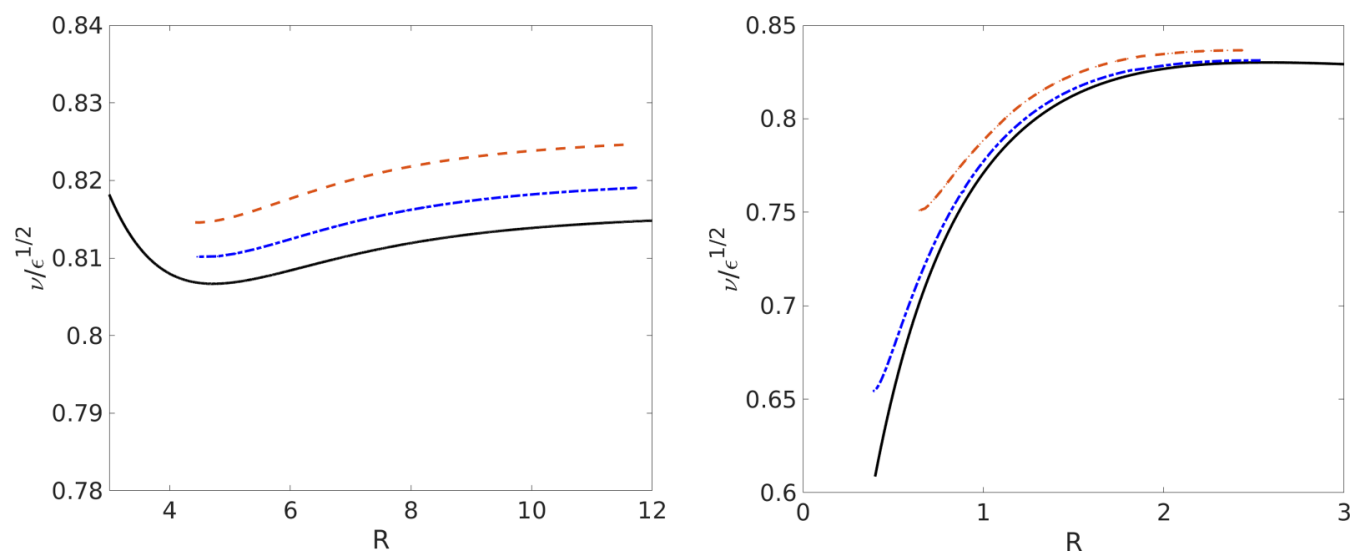

FIG. 8. Comparisons of the analytical prediction [solid (black)] and numerical computations of vesicle equilibria for dimension $d=2$ (left) and $d=3$ (right). The [dashed (red)] curves were computed using $\epsilon=5 \times 10^{-2}$ and the [dash-dotted (blue)] curves were computed using $\epsilon=1.25 \times 10^{-3}$.

expansive view of the relationship between $v_{\infty}$ and volume reveals that competitive instability is very weak for large radii (Fig. 9). In fact, one can show that $v_{\infty} \sim v_{0}+\exp (-C R)$ as $R \rightarrow \infty$. Ripening induced by the exponentially small correction to the large-radius chemical potential $v_{0}$ is likely irrelevant over experimental timescales.

Small spherical vesicles may be either stable or unstable to competition. This is an unexpected prediction of the theory and highlights the significant role that finite width of the curved bilayer plays. Competitive stability of small structures is not typically expected when surface energy alone is taken into account, but the energy of polymer stretching might be a dominant factor when the bilayer width becomes comparable to the radius.

\section{STABILITY OF EQUILIBRIA}

Physical realization of various amphiphilic morphologies relies in part on robustness to both localized shape fluctuations as well as the ability to coexist with neighboring structures. One way of characterizing insensitivity to perturbations is through linearized stability of equilibria arising from the dynamics (6). Note that the (somewhat arbitrarily) prescribed kinetics is used simply as a convenient means to compute linear growth rates, and the qualitative aspects of stability are solely determined by the variational structure of the free energy (3).

It is straightforward to study stability in the context of the approximation scheme used to construct equilibria. This amounts to deriving a free boundary problem for the interfaces, denoted collectively as $\partial \Omega$, enclosing the hydrophobic region $\Omega$. Details of this approximation are given in Appendix B. It is found that the interface motion is governed by a nonlocal free boundary problem having the form

$$
\begin{gathered}
\Delta V=(1-f) \chi_{\Omega}, \quad \boldsymbol{X} \notin \partial \Omega, \\
V=-\sigma \kappa, \quad \boldsymbol{X} \in \partial \Omega, \\
v_{n}=[\partial V / \partial n] .
\end{gathered}
$$

Here $\kappa$ is the mean interface curvature, $\chi_{\Omega}$ is the characteristic function of the set $\Omega$, and [] refers to the jump across the

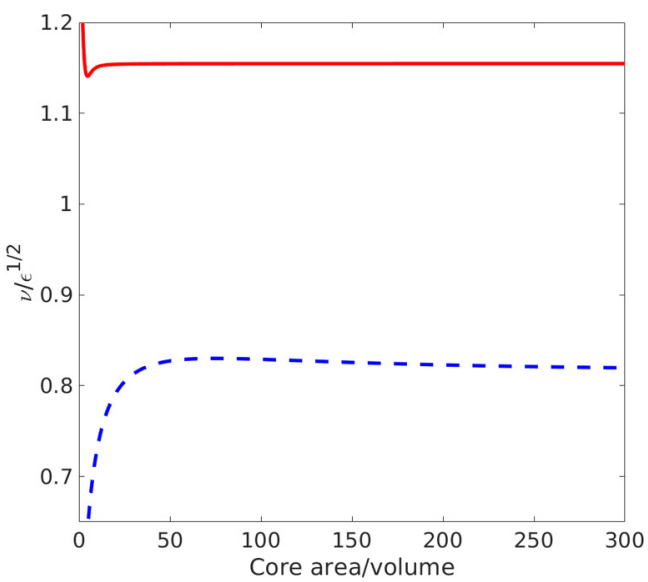

FIG. 9. Left: dependence of the core width as a function of radius for two-dimensional cylindrical vesicles [red (solid)] and spherical vesicles [blue (dashed)]. Right: Behavior of the ambient chemical potential as a function of structure volume for two-dimensional cylindrical vesicles [red (solid)] and spherical vesicles [blue (dashed)]. 
interface oriented in the prescribed normal direction. The curvature is defined so that it is negative when $\Omega$ is convex, and the interface velocity $v_{n}$ is positive when motion is in the direction of the outward normal to $\partial \Omega$. This formulation combines the chemical potentials $v$ and $\mu$ into a single field $V$, which depends on a mesoscale coordinate $\boldsymbol{X}=(X, Y, Z)=$ $\boldsymbol{x} / \epsilon^{1 / 3}$. In addition to (17)-(19), there are far-field conditions appropriate to the geometry under consideration.

This section considers the linearized stability of (17)-(19) for some of the configurations in Sec. III. Remarkably closedform expressions for linearized growth rates and modes of instability can be derived. Implications for morphological preference are discussed later in Sec. V.

\section{A. Bilayers}

Here transverse perturbations to a flat bilayer sheet are considered, by regarding interfaces as graphs $X= \pm w_{0}+$ $w_{ \pm}(Y, Z, t)$ with $w_{ \pm} \ll 1$, where $w_{0}$ is the equilibrium thickness in scaled variables. The linearized evolution for $w_{ \pm}$ satisfies

$$
\begin{gathered}
\Delta V=0, \quad|X| \neq w_{0}, \\
V\left( \pm w_{0}\right)=-\sigma \kappa-(1-f)^{2} w_{0} w_{ \pm}, \\
\lim _{|X| \rightarrow \infty} V_{X}=0, \\
\frac{\partial w_{ \pm}}{\partial t}= \pm\left[V_{X}\right]-(1-f)^{2} w_{ \pm},
\end{gathered}
$$

where the linearized curvatures are $\kappa=\partial^{2} w_{ \pm} / \partial Y^{2}+$ $\partial^{2} w_{ \pm} / \partial Z^{2}$. form

It suffices to consider solutions to the linear system of the

$$
\left(\begin{array}{l}
w_{+} \\
w_{-}
\end{array}\right)=e^{\lambda t+i k_{y} Y+k_{z} Z} \boldsymbol{a}, \quad \boldsymbol{a} \equiv\left(\begin{array}{l}
a_{1} \\
a_{2}
\end{array}\right) .
$$

The corresponding perturbed field is

$$
\begin{aligned}
& V=e^{\lambda t+i k_{y} Y+k_{z} Z}\left[\sigma k^{2}-(1-f)^{2} w_{0}\right] \\
& \times \begin{cases}a_{1} \exp \left[-k\left(X-w_{0}\right)\right], & X>w_{0} \\
a_{2} \exp \left[k\left(X+w_{0}\right)\right], & X<-w_{0} . \\
a_{1} \frac{\sinh \left[k\left(X+w_{0}\right)\right]}{\sinh \left(2 k w_{0}\right)}+a_{2} \frac{\sinh \left[k\left(X-w_{0}\right)\right]}{\sinh \left(-2 k w_{0}\right)}, & |X|<w_{0}\end{cases}
\end{aligned}
$$

where $k=|\boldsymbol{k}|$. Inserting this into the interface dynamics equation (23) yields an eigenvalue problem

$$
\begin{aligned}
\boldsymbol{M a}= & \lambda \boldsymbol{a}, \boldsymbol{M}=\left[\sigma k^{2}-(1-f)^{2} w_{0}\right] \\
& \times\left(\begin{array}{cc}
-k\left[1+\operatorname{coth}\left(2 w_{0} k\right)\right] & k \csc \left(2 w_{0} k\right) \\
k \csc \left(2 w_{0} k\right) & -k\left[1+k \operatorname{coth}\left(2 w_{0} k\right)\right]
\end{array}\right) \\
& -(1-f)^{2} \boldsymbol{I} .
\end{aligned}
$$

The two scaled eigenvalues $\lambda_{ \pm}=\lambda /(1-f)$ may be found explicitly in terms of the dimensionless wave number $q=$ $k w_{0}$, giving

$$
\begin{aligned}
\lambda_{ \pm}= & -q\left(\Sigma q^{2}-1\right)\left[1+\frac{\cosh (2 q) \pm 1}{\sinh (2 q)}\right]-1, \\
& \times \Sigma \equiv \frac{\sigma}{w_{0}^{3}(1-f)^{2}}
\end{aligned}
$$

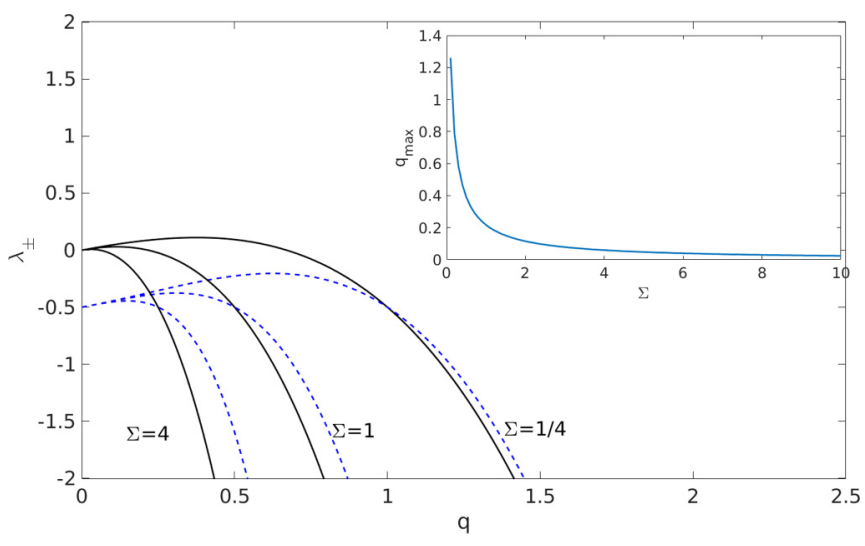

FIG. 10. Linearized growth rates of the bilayer evolution as a function of dimensionless wave number $q\left[\lambda_{+}\right.$are solid (black) curves, corresponding to sinuous modes; $\lambda_{-}$are dashed (blue), corresponding to varicose modes]. Inset: Dependence of the neutrally stable wave number as a function of dimensionless surface tension.

where the corresponding eigenvectors are

$$
\boldsymbol{a}_{+}=\left(\begin{array}{c}
1 \\
-1
\end{array}\right), \quad \boldsymbol{a}_{-}=\left(\begin{array}{l}
1 \\
1
\end{array}\right)
$$

The mode given by $\boldsymbol{a}_{+}$represents a sinuous perturbation, with interfaces moving in the same direction, whereas $\boldsymbol{a}_{-}$ represents a varicose perturbation, with the interfaces moving in opposite directions. Note that $\lambda_{+}(q=0)=0$ and $d \lambda_{+} / d q(q=0)>0$; therefore a bilayer is always unstable for large wavelength perturbations.

It can be shown that the sinuous instability is always dominant. For $\Sigma q^{2}-1<0$, it is easy to see that $\lambda_{+}>\lambda_{-}$. On the other hand, if $\Sigma q^{2}-1>0$, both $d \lambda_{+} / d q$ and $d \lambda_{-} / d q$ are negative. Therefore $\max _{q} \lambda_{+}(q)>\max _{q} \lambda_{-}(q)$. This suggests that bending is the primary dynamic phenomenon associated with bilayers sheets.

Figure 10 illustrates the dependence of eigenvalues on wave number, as well as the wave number of the neutral mode as a function of dimensionless surface energy. The propensity to bend decreases with either increasing surface tension or decreasing bilayer width.

The foregoing calculation must be viewed with caution. The bilayer width $w_{0}$ is measured on a scale relative to $\epsilon^{1 / 3}$, whereas competitively stable bilayers may have widths down to a scale $\sim \epsilon \ln \epsilon$. Narrow bilayers correspond to large $\Sigma$, and in this case the wavelength for instability is pushed off to infinity. Therefore the sinuous instability reported has relevance only for relatively thick bilayers. There is a simple physical interpretation: a flat bilayer which is hypothetically stretched in the normal direction can relieve strain by buckling, which has the effect of reducing the cross-sectional width.

\section{B. Cylindrical micelles}

Radially symmetric equilibria for dimension $d=2$ correspond to cylindrical micelles of infinite length. Stability may be studied in a fashion similar to the previous section, by deriving the linearized dynamics of a perturbed cylindrical interface described by a graph $\rho=R_{0}+R(Y, \theta, t)$. Here 

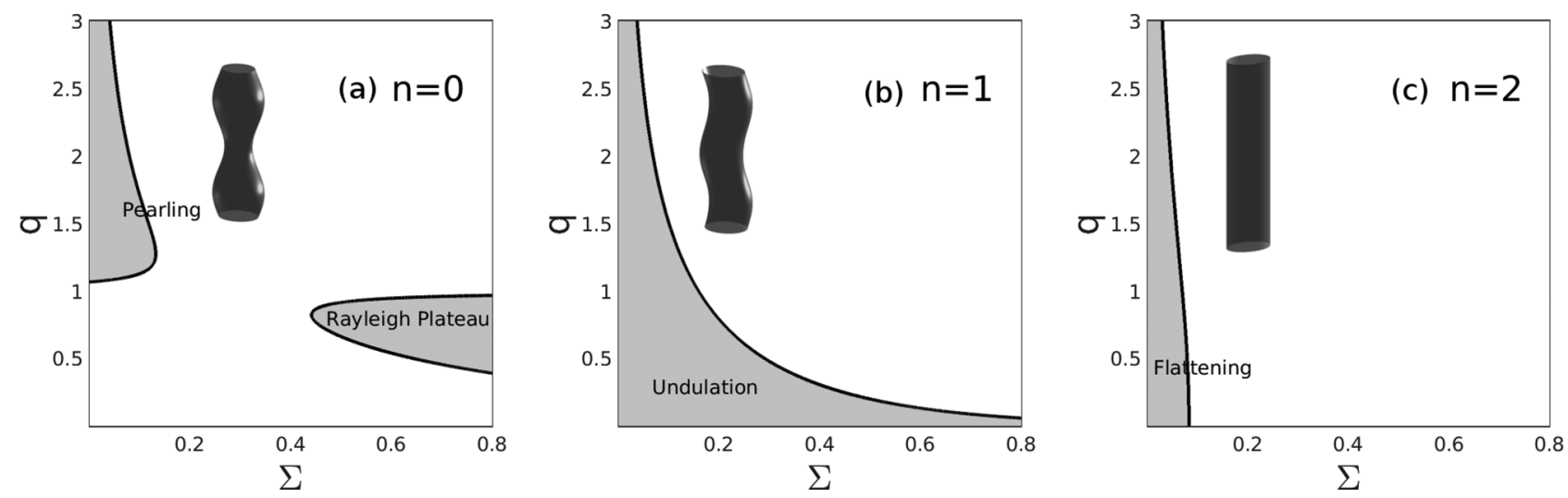

FIG. 11. Regions of instability of cylindrical micelles. Here $\Sigma$ is the dimensionless surface tension, $q$ the reduced wave number, and $n$ is the azimuthal mode. Two types of axisymmetric instabilities are identified (a), a small wavelength pearling instability and a large wavelength Rayleigh-Plateau instability. The $n=1$ mode (b) reveals an undulational long wavelength instability. For small $\Sigma$, there is also a flattening instability (c).

$(\rho, \theta, Y)$ are scaled cylindrical coordinates, and $R_{0}$ is the scaled equilibrium radius.

The linearized dynamics for $R \ll 1$ obeys

$$
\begin{gathered}
\Delta V=0, \quad \rho \neq R_{0}, \\
V=-\sigma\left(R / R_{0}^{2}+R_{\rho \rho}+R_{\theta \theta} / R_{0}^{2}\right) \\
-(1-f)^{2} R_{0} R / 2, \\
\rho=R_{0}, \\
\lim _{\rho \rightarrow \infty}\left(V-\rho V_{\rho} \ln \rho\right)=-\ln (2 \sqrt{\beta}) \rho V_{\rho}, \\
\frac{\partial R}{\partial t}=\left[V_{\rho}\right]-(1-f)^{2} R .
\end{gathered}
$$

With $R=\exp (\lambda t+i k Y+i n \theta)$, where $n=1,2,3, \ldots$ the field solution is

$$
\begin{aligned}
V= & e^{\lambda t+i k Y+i n \theta}\left[\sigma\left(k^{2}+n^{2} / R_{0}^{2}-1 / R_{0}^{2}\right)-(1-f)^{2} R_{0} / 2\right] \\
& \times\left\{\begin{array}{ll}
K_{n}(k \rho) / K_{n}\left(k R_{0}\right), & \rho>R_{0} \\
I_{n}(k \rho) / I_{n}\left(k R_{0}\right), & \rho<R_{0}
\end{array} .\right.
\end{aligned}
$$

Here $K_{n}, I_{n}$ are Bessel functions of order $n$. Condition (31) is specialized to the geometry under consideration; see Eq. (B18). Equation (32) implies the scaled growth rate in terms of the nondimensional wave number $q=k R_{0}$ is

$$
\begin{aligned}
\lambda & =q\left[\Sigma\left(q^{2}+n^{2}-1\right)-1 / 2\right]\left[\frac{K_{n}^{\prime}(q)}{K_{n}(q)}-\frac{I_{n}^{\prime}(q)}{I_{n}(q)}\right]-1, \\
\Sigma & \equiv \frac{\sigma}{R_{0}^{3}(1-f)^{2}} .
\end{aligned}
$$

Figure 11 displays regions of instability in the $\Sigma, q$ plane. For axisymmetric disturbances, there are two types, associated with short and long wavelengths. When $\Sigma$ is small enough, there is a pearling-type instability (e.g., Ref. [44]). For large $\Sigma$, there is another unstable region, which is simply the extension of the Rayleigh-Plateau instability, which would be expected when surface tension dominates (i.e. $\Sigma \rightarrow$ $\infty)$. Note that there is an intermediate range $0.15<\Sigma<0.46$ where no axisymmetric instability is found. The azimuthal $n=1$ mode corresponds to undulations which preserve the cylindrical cross section. As in the case of bilayers, instability at long wavelengths is always present. Finally, unstable modes which represent flattening of the cylinder $(n=2)$ may be present for small $\Sigma$, but these are always subdominant compared to the undulation modes.

The case where $k=0=n$ merits special consideration. The field solution is

$$
V=-e^{\lambda t}\left(\sigma / R_{0}^{2}\right)\left\{\begin{array}{ll}
1 & \rho>R_{0} \\
A \ln \left(\rho / R_{0}\right)+1 & \rho<R_{0}
\end{array},\right.
$$

where by (31), one has $A=-1 / \ln \left(R_{0} / 2 \sqrt{\beta}\right)$. Then

$$
\lambda=-\sigma A / R_{0}^{3}-(1-f)^{2} .
$$

The value of $R_{0}$ corresponding to $\lambda=0$ is the threshold for competitive stability and is precisely the same (up to asymptotic corrections) as predicted by the minimum in (12).

The above results should be carefully interpreted in the context of cylindrical micelles of finite extent, which have end caps that have not been accounted for. Nevertheless, it can be expected that finite cylinder length could suppress long wavelength instabilities. On the other hand, it is reasonable that short wavelength pearling-type instabilities can persist for long but finite length structures.

\section{Spherical micelles}

Perturbation of a spherical equilibrium interface of radius $R_{0}$ can be described by a graph $\rho=R_{0}+R(\theta, \phi, t)$, where $(\theta, \phi)$ are azimuthal coordinates. The corresponding linearized evolution is resulting in

$$
\begin{gathered}
\Delta V=0, \quad \rho \neq R_{0}, \\
V=-\sigma\left(\Delta_{s} R+2\right) / R_{0}^{2}-(1-f)^{2} R_{0} R / 3, \quad \rho=R_{0}, \\
\lim _{\rho \rightarrow \infty} V=0, \\
\frac{\partial R}{\partial t}=\left[V_{\rho}\right]-(1-f)^{2} R .
\end{gathered}
$$

Here $\Delta_{s}$ is the spherical surface (Laplace-Beltrami) operator. 


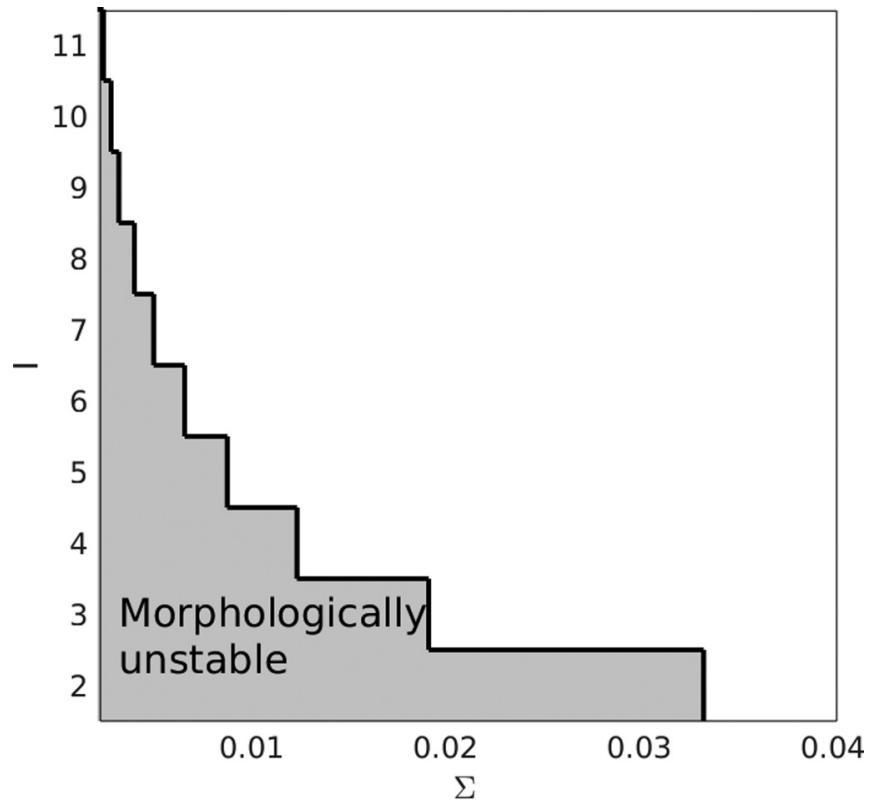

FIG. 12. Region of instability for spherical micelles in terms of the spherical harmonic index $l$ and the dimensionless surface tension $\Sigma$.

It suffices to consider perturbations of the form $R=$ $\exp (\lambda t) Y_{l}^{m}(\theta, \phi)$, where $Y_{l}^{m}$ is a spherical harmonic. The resulting field solution is

$$
\begin{aligned}
V= & e^{\lambda t} Y_{l}^{m}(\theta, \phi)\left\{\sigma[l(l+1)-2] / R_{0}^{2}-(1-f)^{2} R_{0} / 3\right\} \\
& \times \begin{cases}\rho^{l} / R_{0}^{l}, & \rho<R_{0} \\
\rho^{-l-1} / R_{0}^{-l-1}, & \rho>R_{0}\end{cases}
\end{aligned}
$$

leading to a scaled growth rate

$$
\lambda=-(2 l+1)\{\Sigma[l(l+1)-2]-1 / 3\}-1,
$$

where the dimensionless surface tension $\Sigma$ is the same as in (34).

The region for instability as a function of dimensionless surface tension is shown in Fig. 12. The modes $l>2$ exhibit instability for small enough surface tension, whereas for $\Sigma>$ $1 / 30$, they are all stable. The mode $l=1$ corresponds to translation and is therefore neutral. Competitive stability is determined by the $l=0$ mode, which occurs when $\Sigma>1 / 3$, coinciding with the minima of $v_{\infty}(R)$ in Fig. 12.

\section{MORPHOLOGICAL PREFERENCE}

Localized structures may form spontaneously under the dynamics given by (6) [26,33]. Many phenomenon influence the subsequent relaxation toward global equilibrium, including mass exchange, nonlocal interactions that drive rigidbody motion, and coalescence events [31]. The dominant mechanism for well-separated structures is diffusion of material driven by differences in polymer chemical potential $v$ (whereas $\mu$ is associated with compositional differences within the polymer phase). Thus equilibrium requires that the ambient chemical potential $v_{\infty}$ associated to each structure should be the same. This does not, however, preclude the possibility of coexistence of different geometries classes.

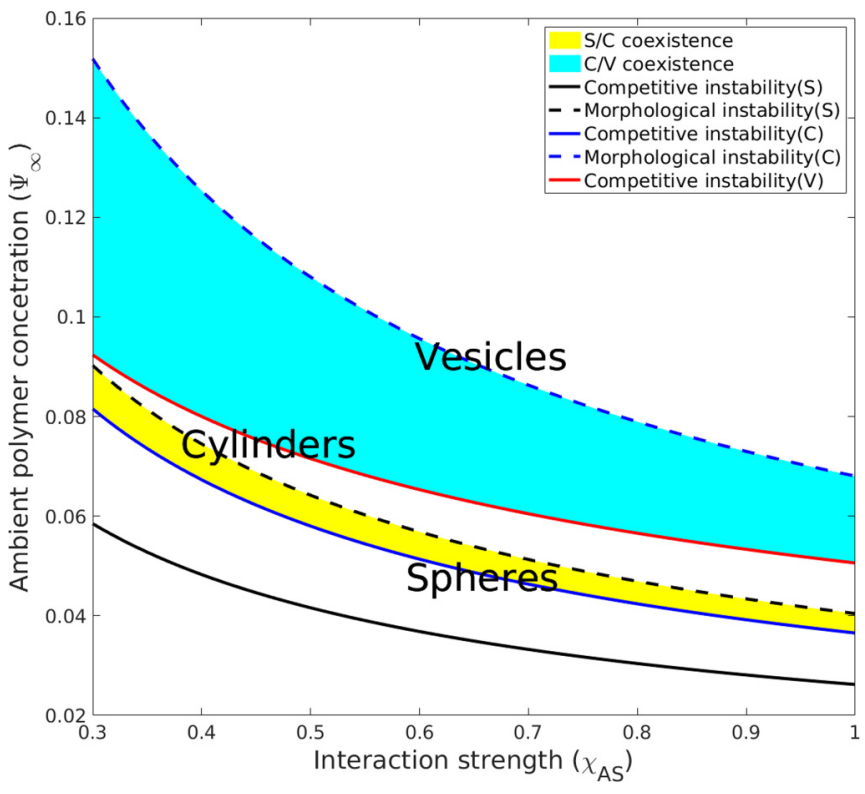

FIG. 13. Regions of stability for spherical and cylindrical micelles, and spherical vesicles. These regions overlap, indicating the possibility of coexistence.

Experimental observations [9] in fact note the persistence of multiple morphological populations over very long times. On the other hand, both competitive and morphological stability is required for individual elements to remain intact.

Both composition and molecular interaction strength have been shown to influence the preference toward either spherical, cylindrical, or vesicle phases in synthetic amphiphilic systems. Solvent or hydrophobic interactions are measured by $\chi_{A S}$, which ultimately controls the surface tension parameter $\sigma$. For potential (5), for example, one finds that $\sigma=\chi_{A S}^{1 / 2} / 6$. The equilibrium composition of polymer in the bulk solvent $\Psi_{\infty}$ is related to the ambient chemical potential $v_{\infty}$ via (8). For potential (5) and small compositions, one finds $v_{\infty} \approx$ $2 \chi_{A S} \Psi_{\infty}$.

For given values of $\chi_{A S}$ and $\Psi_{\infty}$, a particular type of equilibrium may or may not exist, depending on whether $v_{\infty}$ sits below the $v_{\infty}(R)$ curve (see, for example, Fig. 5). In addition, even in the case of existence, both morphological and competitive instabilities might drive a localized domain structure toward extinction or changes in topology. Note that the threshold for competitive stability coincides with the fold bifurcation, so it is sufficient to characterize viability of any morphology in terms of stability alone. As either $\chi_{A S}$ or $\Psi_{\infty}$ is increased, the threshold for morphological instability is then crossed. The window between the two curves that define stability thresholds produces a region of parameter space where a particular morphology may be observed.

Regions of stability in the $\left(\chi_{A S}, \Psi_{\infty}\right)$ parameter space were computed for spherical and cylindrical micelles, as well as spherical vesicles, using potential (5). Figure 13 summarizes the results. As either the polymer concentration or interaction strength is increased, preference shifts from spheres to cylinders to vesicles. On the other hand, there are significant overlapping domains between spherical and cylindrical micelles, as well as cylinders and vesicles. In these parameter 
regions, coexistence of multiple morphological populations is possible. The bifurcation sequence leading from spheres to cylinders to vesicles has been reported in numerical studies $[6,22,32]$.

For the cylindrical case, morphological stability in Fig. 13 reflects only the effect of short-wavelength perturbations, i.e., those associated with the pearling region in Fig. 11. This choice reflects the finite size expected in real systems, where only rodlike micelles are observed in practice $[7,8]$. Another complication of the cylindrical case is that there is an additional dimensionless quantity $R / \sqrt{\beta}$ [see the first expression in (12)]. For the purposes of Fig. 13, this ratio was arbitrarily chosen to be $1 / 2$; other choices over a range of values from $1 / 10$ to 1 appear to give a qualitatively (and nearly quantitatively) similar picture.

\section{DISCUSSION AND CONCLUSION}

Density functional models for amphiphilic systems provide a valuable connection between molecular scale physics and amphiphilic morphology. This was done here by exploiting the separation of length scales between phase domains and interfaces through the use of multiscale analyses. This limit is analogous to the strong segregation regime of block copolymer mixtures (e.g., Ref. [45]), but there appears to be no similar theory for SCFT models of amphiphilic systems. The benefit of the density functional model approach is that the sharp interface regime is readily accessible and leads to explicit formulas connecting the structure size to the ambient chemical potential. In addition, the parameters in these formulas $(\beta, \sigma, \gamma, \lambda)$ can be directly related to the continuum model and have a straightforward physical interpretation in terms of length scales and thermodynamic quantities, making comparison to experiment possible.

\section{A. Comparison to experimental phase diagrams}

Our analytic results immediately lead to prediction of morphological preference as summarized in the phase diagram. This allows for coexistence of different stable morphologies, consistent with experimental findings [7,9,46,47]. Our conclusion differs from the study of a phenomenological model by Christlieb et al. [48], who find that bilayers (in the form of vesicles) and cylindrical micelles cannot coexist over long times. Jain and Bates [9] suggest that coexistence is largely a result of slow kinetics, and that global equilibrium is inaccessible on experimental timescales. In contrast, we have shown that coexistence is possible regardless of kinetics, since competitive stability may be achieved simultaneously among different types of equilibria.

\section{B. Comparison to SCFT calculations}

He and Schmid [22] used extensive numerical calculation of a SCFT model to assemble a diagram of observed equilibrium morphologies as a function of polymer concentration and solvent-hydrophilic Flory-Huggins parameter $\chi_{B S}$. It is unclear how their results would vary as a function of the four other model parameters that were fixed in their calculation, so a quantitative comparison with this work is not readily available. Interestingly, Ref. [22] found structure types not considered here, specifically cage-type vesicles and toroidal micelles. Numerical simulations of the density functional model suggest these can be studied in the present framework; this would be a valuable route for future study.

\section{Relation to the Canham-Helfrich membrane energy}

A commonly utilized model for the mechanics of amphiphilic bilayers was introduced by Canham [49] and Helfrich [50](called $\mathrm{CH}$ hereafter). This model treats a bilayer as a thin elastic surface with no internal structure, which of course excludes micelle architectures altogether. In their most basic form, the $\mathrm{CH}$ model proposes a free energy of the form $\int \kappa^{2} d A$, where the integral is over the two-dimensional bilayer surface. In the absence of other constraints, a flat bilayer sheet would therefore be entirely stable.

Both our model and the $\mathrm{CH}$ formalism predict sphericalshaped vesicles [43]. In the latter case, it is easy to see that the free energy is completely independent of vesicle radius, which means that there is no propensity for competitive instability. As mentioned in Sec. III C, our results indicate that competitive instability becomes exceedingly weak for large vesicles. In this respect, our results are consistent with the $\mathrm{CH}$ predictions and should be viewed as an interpolation between macroscopic behavior (as described by $\mathrm{CH}$ ) and smaller scale phenomena.

\section{Relation to instability of other cylindrical structures}

In the classical Rayleigh-Plateau problem [51], surface energy alone is responsible for instability of the free surface, which always exists at long wavelengths. Here the polymer contribution to the free energy appears to suppress this instability over a range of dimensionless surface tension $\Sigma$ but also enhances other modes of instability. The suppression and/or modification of the Rayleigh-Plateau instability is not a feature unique to amphiphiles; recent studies of capillary instabilities in gels $[52,53]$ highlight the stabilizing role played by elastic forces.

If the surface tension contribution is insufficient, axisymmetric short wavelength instabilities may arise, leading to pearling behavior. This phenomenon has also been studied in the context of phenomenological models of amphiphiles [54] and polymer brushes [55]. Pearling phenomenon have also been observed in biological and synthetic membranes [44,56].

\section{E. Outlook}

Beyond issues of basic equilibrium shapes and stability, one wonders what the effective elastic energy of a curved bilayer might be in our model. One possible scenario is that some variant of the $\mathrm{CH}$ energy can be recovered in a certain distinguished limit, as has been done for certain phenomenological models [57-59]. This would likely require further expansion terms which were not considered in this work.

We utilized linear (dynamic) stability as a criteria for realizability of specific geometries. This is an incomplete classification, because instabilities may lead to far more exotic forms that have been reported experimentally [8]. In this respect, morphological selection should be regarded as both 
a function of system parameters as well as initial conditions. A more complete assessment of dynamic pathways leading to equilibria is needed to fully characterize the accessible range of self-assembled architectures.

\section{ACKNOWLEDGMENT}

The author was supported through NSF Awards No. DMS1514689 and No. DMS-1908968.

\section{APPENDIX A: MATCHED ASYMPTOTIC EXPANSIONS}

\section{Bilayers}

It is assumed that $\nu_{\infty}(\epsilon)=o(1)$ as $\epsilon \rightarrow 0$, but otherwise the dependence on $\epsilon$ is not initially prescribed; rather, it will ultimately be determined by a nonlinear relationship with the scaled bilayer half-width $w(\epsilon)$. Stretched coordinates $z=x / \epsilon$ and $\zeta=z-w$ are used in regions I and II, respectively.

The leading order solutions $\Phi_{0}, \Psi_{0}$ in each region are

$$
\left(\begin{array}{c}
\Phi_{0} \\
\Psi_{0}
\end{array}\right)= \begin{cases}\left(\begin{array}{c}
1-f \\
f
\end{array}\right), & \text { Region I } \\
\left(\begin{array}{l}
\Phi_{h} \\
\Psi_{h}
\end{array}\right), & \text { Region II } \\
\left(\begin{array}{l}
0 \\
0
\end{array}\right), & \text { Region III }\end{cases}
$$

where the region II solution is the heteroclinic orbit of

$$
-\left(\begin{array}{l}
\Phi_{h} \\
\Psi_{h}
\end{array}\right)_{\zeta \zeta}+\nabla W\left(\Phi_{h}, \Psi_{h}\right)=0
$$

chosen so that its components approach zero when $\zeta \rightarrow$ $\infty$ and $(1-f, f)$ when $\zeta \rightarrow-\infty$. Such a solution exists under the given hypothesis on $W$ (see Ref. [60] and references therein) and is uniquely determined up to translation. Linearization about $(1-f, f)$ establishes the asymptotic behavior

$$
\left(\begin{array}{l}
\Phi_{h} \\
\Psi_{h}
\end{array}\right) \sim\left(\begin{array}{c}
1-f \\
f
\end{array}\right)-\exp ^{\lambda_{1} \zeta} \boldsymbol{a}_{1}-\exp ^{\lambda_{2} \zeta} \boldsymbol{a}_{2}, \quad \zeta \rightarrow-\infty
$$

where $\boldsymbol{a}_{j}, \lambda_{j}>0$ solve the eigenvalue problem

$$
H^{A} \boldsymbol{a}_{j}=\lambda_{j}^{2} \boldsymbol{a}_{j}, \quad H^{A} \equiv \nabla^{2} W(1-f, f) .
$$

For simplicity, we suppose $\lambda_{1}=\lambda_{2}=\lambda$, and the eigenvectors $\boldsymbol{a}_{j}$ are chosen to be orthogonal.

The expansion in region III is written $\Phi \sim \Phi_{1}(x ; \epsilon)+\cdots$, where $\Phi_{1}=o(1)$ as $\epsilon \rightarrow 0$, with a similar expansion for $\Psi$. The dependence on $\epsilon$ is not prescribed a priori but is assumed to be the same asymptotic order as $\nu_{\infty}(\epsilon)$. Similarly expanding in the variables $\mu$ and $v$ leads to

$$
\left(\begin{array}{l}
\mu_{1} \\
\nu_{1}
\end{array}\right)=H^{0}\left(\begin{array}{l}
\Phi_{1} \\
\Psi_{1}
\end{array}\right), \quad H^{0} \equiv \nabla^{2} W(0,0) .
$$

It follows that $v_{1}=v_{\infty}(\epsilon)$, and $\Phi_{1}$ solves the linear problem $\left(H_{\Phi \Phi}^{0} \Phi_{1}+H_{\Phi \Psi}^{0} \Psi_{1}\right)_{x x}=\Phi_{1}$ whose solution is

$$
\Phi_{1}=\Phi_{1}(x=0) \exp (-x / \sqrt{\beta}), \quad \beta \equiv \operatorname{det} H^{0} / H_{\Psi \Psi}^{0} .
$$

For region II, the same expansions for $\Phi$ and $\Psi$ are used, but with independent variable $\zeta$. Since matching implies
$\Phi_{1}(\zeta) \rightarrow \Phi_{1}(x=0)$ as $\zeta \rightarrow \infty$, the equation for $\Phi_{1}$ may be integrated twice to give the linear problem

$$
\mathcal{L}\left(\begin{array}{c}
\Phi_{1} \\
\Psi_{1}
\end{array}\right)=\left(\begin{array}{c}
\bar{\mu} \\
v_{\infty}
\end{array}\right), \quad \mathcal{L} \equiv-\partial_{\zeta \zeta}+\nabla^{2} W\left(\Phi_{h}, \Psi_{h}\right),
$$

where $\bar{\mu}$ is an integration constant, which by matching is

$$
\bar{\mu}=\beta \Phi(x=0)+\frac{H_{\Phi \Psi}^{0}}{H_{\Psi \Psi}^{0}} v_{\infty}(\epsilon)
$$

Solutions to (A7) are bounded as $\zeta \rightarrow \infty$ but must grow exponentially for $\zeta \rightarrow-\infty$ to accommodate matching to region $\mathrm{I}$.

A solvability condition for may be derived by taking the dot product of (A7) with the eigenfunction $\left(\Phi_{h}, \Psi_{h}\right)_{\zeta}$ and integrating from $\zeta$ to $\infty$, resulting in

$$
\begin{aligned}
& -\left(\begin{array}{c}
\Phi_{1} \\
\Psi_{1}
\end{array}\right) \cdot\left(\begin{array}{c}
\Phi_{h} \\
\Psi_{h}
\end{array}\right)_{\zeta \zeta}+\left(\begin{array}{c}
\Phi_{1} \\
\Psi_{1}
\end{array}\right)_{\zeta} \cdot\left(\begin{array}{c}
\Phi_{h} \\
\Psi_{h}
\end{array}\right)_{\zeta} \\
& \sim-\left(\begin{array}{c}
\bar{\mu} \\
v_{\infty}
\end{array}\right) \cdot\left(\begin{array}{c}
1-f \\
f
\end{array}\right), \quad \zeta \rightarrow-\infty .
\end{aligned}
$$

Using (A3), this can be written

$$
\begin{gathered}
\sum_{j=1,2} \exp (\lambda \zeta) \boldsymbol{a}_{j} \cdot\left[\lambda^{2}\left(\begin{array}{l}
\Phi_{1} \\
\Psi_{1}
\end{array}\right)-\lambda\left(\begin{array}{l}
\Phi_{1} \\
\Psi_{1}
\end{array}\right)_{\zeta}\right] \\
\sim-\left(\begin{array}{c}
\bar{\mu} \\
v_{\infty}
\end{array}\right) \cdot\left(\begin{array}{c}
1-f \\
f
\end{array}\right), \quad \zeta \rightarrow-\infty .
\end{gathered}
$$

Anticipating exponential growth for $\zeta \rightarrow-\infty$ of the form

$$
\left(\begin{array}{l}
\Phi_{1} \\
\Psi_{1}
\end{array}\right) \sim \sum_{j=1,2} k_{j} \exp (-\lambda \zeta) \boldsymbol{a}_{j}
$$

it follows that the undetermined coefficients $k_{1,2}$ are related by

$$
2 \lambda^{2}\left(k_{1}+k_{2}\right)=-\left(\begin{array}{c}
\bar{\mu} \\
v_{\infty}
\end{array}\right) \cdot\left(\begin{array}{c}
1-f \\
f
\end{array}\right) .
$$

For region I, expansions $\Phi=1-f+\Phi_{1}(z ; \epsilon)+\cdots$ and $\Psi=f+\Psi_{1}(z ; \epsilon)+\cdots$ are used, but with $\epsilon$ dependence different than the other regions. In particular, matching will require that $\Phi_{1}(z, \epsilon) \gg \Phi_{1}(\zeta, \epsilon)$ with a similar ordering for $\Psi$. After integrating the equation for $\Phi_{1}$ twice and using the reflective boundary conditions at $z=0$, one obtains

$$
-\left(\begin{array}{c}
\Phi_{1} \\
\Psi_{1}
\end{array}\right)_{z z}+H^{A}\left(\begin{array}{l}
\Phi_{1} \\
\Psi_{1}
\end{array}\right)=\left(\begin{array}{l}
C \\
0
\end{array}\right), \quad \Phi_{1 z}(0)=0=\Psi_{1 z}(0)
$$

where $C$ is a (ultimately irrelevant) constant of integration. The solution of (A13) is

$$
\left(\begin{array}{l}
\Phi_{1} \\
\Psi_{1}
\end{array}\right)=\boldsymbol{p}+\sum_{j=1,2} c_{j}(\epsilon)\left(e^{\lambda z}+e^{-\lambda z}\right) \boldsymbol{a}_{j}, \quad H^{A} \boldsymbol{p}=\left(\begin{array}{l}
C \\
0
\end{array}\right)
$$

where constants $c_{j}$ are to be determined.

Expansions in regions I and II may now be matched. Writing the asymptotic solution for $\zeta \rightarrow-\infty$ in region II in 
terms of the variable $z$ in (A3) and (A11), one has

$$
\left(\begin{array}{l}
\Phi_{1} \\
\Psi_{1}
\end{array}\right) \sim\left(\begin{array}{c}
1-f \\
f
\end{array}\right)+\sum_{j=1,2}\left(k_{j} e^{\lambda w(\epsilon)} e^{-\lambda z}-e^{-\lambda w(\epsilon)} e^{\lambda z}\right) \boldsymbol{a}_{j}
$$

Equating coefficients to those in (A14) gives

$$
c_{1}(\epsilon)=-e^{-\lambda w(\epsilon)}=c_{2}(\epsilon)=k_{1}(\epsilon) e^{\lambda w(\epsilon)}=k_{2}(\epsilon) e^{\lambda w(\epsilon)} .
$$

Together with (A12) this implies

$$
4 \lambda^{2} e^{-2 \lambda w(\epsilon)}=\left(\begin{array}{c}
\bar{\mu} \\
v_{\infty}
\end{array}\right) \cdot\left(\begin{array}{c}
1-f \\
f
\end{array}\right) .
$$

Further expanding the variable $\mu \equiv-\Phi_{z z}+W_{\Phi}$ in region I using $\mu=-\Phi_{1 z z}+H_{\Phi \Phi}^{A} \Phi_{1}+H_{\Phi \Psi}^{A} \Psi_{1}+\epsilon^{2} \mu_{*}+o\left(\epsilon^{2}\right)$, one obtains

$$
\mu_{* z z}=1-f, \quad \mu_{* z}(0)=0,
$$

which integrates to $\mu_{* z}=(1-f) z$. This matches to an expansion term $\mu_{* \zeta}(\zeta)$ in region II with order $\epsilon^{2} w(\epsilon)$, satisfying $\mu_{* \zeta \zeta}=0$, which means that $\mu_{* \zeta}$ is constant throughout region II. This in turn matches the leading order expression of the expansion for $\mu_{x}$ in region III. The latter is $\mu_{1 x}=H_{\Phi \Phi}^{0} \Phi_{1}+$ $H_{\Phi \Psi}^{0} \Psi_{1}$, and it follows that

$$
\mu_{1 x}(x=0)=(1-f) \epsilon w(\epsilon) .
$$

Expressions (A5), (A6), (A8), (A17), and (A18) can be used to eliminate all unknowns except $\nu_{\infty}(\epsilon)$ and $w(\epsilon)$, resulting in (11). A balance of the three terms implicitly defines the orders for expansion terms $\Phi_{1}, \Psi_{1}$. As $w(\epsilon)$ becomes large, the balance is between the last two terms in (11), and one has $v_{\infty} \propto \epsilon w(\epsilon)$.

\section{Micelles}

Here it is assumed that $v_{\infty}, R \ll 1$ for $\epsilon \rightarrow 0$. Regions for radially symmetric micelles are as in Fig. 3, where region I uses a scaled coordinate $\rho=r / R(\epsilon)$ and region II uses $\zeta=$ $(r-R(\epsilon)) / \epsilon$.

It is useful to work in the variable $\mu \equiv-\epsilon^{2} \Delta_{r} \Phi+W_{\Phi}$. In region III this is expanded as $\mu=\mu_{*}(r ; \epsilon)+\mu_{* *}(r ; \epsilon)+\cdots$, where $\mu_{*}=O\left(v_{\infty}(\epsilon)\right), \mu_{* *} \ll \mu_{*}$, and the dependence on $\epsilon$ is determined by matching. A similar expansion is required for $\Psi$, where by contrast, the far-field conditions require the expansion $\Phi \sim \Phi_{* *}(r ; \epsilon)$ with $\Phi_{* *}=O\left(\mu_{* *}\right)$.

Since $\Delta_{r} \mu_{*}=0$ for $r>0$, the solution is just a constant, determined by expansion of the far-field condition

$$
\mu_{*}=\mu_{\infty} \equiv-\frac{H_{\Phi \Psi}^{0}}{H_{\Psi \Psi}^{0}} v_{\infty}
$$

At next order, one finds $\Delta_{r} \mu_{* *}=\Phi_{* *}=\mu_{* *} / \beta$, where $\beta$ is defined as before. The solution can be written

$$
\mu_{* *}=C(\epsilon)\left\{\begin{array}{ll}
K_{0}(r / \sqrt{\beta}), & d=2 \\
\exp (-r / \sqrt{\beta}) / r, & d=3
\end{array} .\right.
$$

For region II, the leading order solutions are the same as in the bilayer case. The second expansion terms $\Phi_{*}(\zeta ; \epsilon), \Psi_{*}(\zeta ; \epsilon)$ are of the same order as $\nu_{\infty}(\epsilon)$ and solve

$$
\mathcal{L}\left(\begin{array}{c}
\Phi_{*} \\
\Psi_{*}
\end{array}\right)-\frac{\epsilon(d-1)}{R(\epsilon)}\left(\begin{array}{c}
\Phi_{h} \\
\Psi_{h}
\end{array}\right)_{\zeta}=\left(\begin{array}{c}
\bar{\mu} \\
v_{\infty}
\end{array}\right)
$$

where $\bar{\mu}$ is an integration constant. We are primarily interested in the regime where $\epsilon / R(\epsilon) \sim \nu_{\infty}(\epsilon)$, so the second term in (A20) is retained. Solvability is determined as before, giving

$$
\begin{aligned}
\frac{\sigma(d-1) \epsilon}{R(\epsilon)} & =\left(\begin{array}{c}
\bar{\mu} \\
v_{\infty}
\end{array}\right) \cdot\left(\begin{array}{c}
1-f \\
f
\end{array}\right) \\
\sigma & \equiv \int_{-\infty}^{\infty}\left(\Phi_{h}^{\prime}\right)^{2}+\left(\Psi_{h}^{\prime}\right)^{2} d \zeta
\end{aligned}
$$

Matching solutions in regions III and II can be accomplished using $\mu_{* *} \sim-C(\epsilon) \ln [R(\epsilon) / 2 \sqrt{\beta}]$ for $d=2$ and $\mu_{* *} \sim-C(\epsilon) / R(\epsilon)$ for $d=3$. It follows that $\mu_{* *}(r)=$ $O\left[\mu_{*}(r) /|\ln \epsilon|\right]$ in dimension 2 and $\mu_{* *}(r)=O\left[R(\epsilon) \mu_{*}\right]$ in dimension 3 , and

$$
\bar{\mu}=\mu_{\infty}+C(\epsilon)\left\{\begin{array}{ll}
-\ln [R(\epsilon) / 2 \sqrt{\beta}], & d=2 \\
1 / R(\epsilon), & d=3
\end{array} .\right.
$$

Defining the variable $J \equiv r^{d-1} \mu_{r}$, the leading order expression in region III has

$$
J \sim r^{d-1} \mu_{2 r} \sim-C(\epsilon), \quad r \rightarrow 0 .
$$

In region I, the leading order expression $J=R(\epsilon)^{d-2} \rho^{d-1} \mu_{\rho}$ solves

$$
\frac{1}{\rho^{d-2}} J_{\rho}=R(\epsilon)^{d}(1-f)
$$

subject to $J(0)=0$, so that

$$
J \sim \frac{(1-f) R(\epsilon)^{d}}{d}, \quad \rho \rightarrow 1 .
$$

Similar to the one-dimensional case, one finds that $J$ is constant in region II to leading order, therefore matching across all three regions leads to

$$
C(\epsilon)=\frac{(1-f) R(\epsilon)^{d}}{d} .
$$

Combining expressions (A21), (A22), and (A26) give the desired relationship (12).

\section{Vesicles}

Region I (Fig. 7) uses a coordinate $\rho=(r-R) / \epsilon^{1 / 2}$, where $R$ represents the midpoint radius. Regions $\mathrm{II}_{ \pm}$use the coordinate

$$
\zeta=\frac{r-R \pm \epsilon^{1 / 2} w}{\epsilon},
$$

where $w$ characterizes the width of region II and is also to be determined. The scaling $\nu_{\infty}=O\left(\epsilon^{1 / 2}\right)$ is prescribed in advance, and all quantities are expanded in powers of $\epsilon^{1 / 2}$.

In the regions $\mathrm{III}_{ \pm}$, one finds that

$$
\left(\begin{array}{c}
\mu_{1 / 2} \\
v_{\infty}
\end{array}\right)=H^{0}\left(\begin{array}{c}
\Phi_{1 / 2} \\
\Psi_{1 / 2}
\end{array}\right),
$$

which may be inverted to write the leading order problem as

$$
\Delta_{r} \mu_{1 / 2}=\frac{1}{\beta}\left(\mu_{1 / 2}-\frac{H_{\Phi \Psi}^{0}}{H_{\Psi \Psi}^{0}} v_{\infty}\right),
$$


where $H^{0}$ and $\beta$ are defined above. The solution in region III with condition (9) is

$$
\mu_{1 / 2}=v_{\infty} \frac{H_{\Phi \Psi}^{0}}{H_{\Psi \Psi}^{0}}+c_{-}\left\{\begin{array}{ll}
I_{0}(r / \sqrt{\beta}), & d=2 \\
\sinh (r / \sqrt{\beta}) / r, & d=3
\end{array},\right.
$$

where $I_{0}$ is a modified Bessel function and $c_{-}$is presently unknown. The decaying solution in region $\mathrm{III}_{+}$is similarly

$$
\mu_{1 / 2}=v_{\infty} \frac{H_{\Phi \Psi}^{0}}{H_{\Psi \Psi}^{0}}+c_{+}\left\{\begin{array}{ll}
K_{0}(r / \sqrt{\beta}), & d=2 \\
\exp (-r / \sqrt{\beta}) / r, & d=3
\end{array} .\right.
$$

For region $\mathrm{II}_{+}$, the leading order is the same as in the onedimensional situation, whereas in region II $_{-}$the direction is reversed so $\Phi_{0}=\Phi_{h}(-\zeta)$ etc. At the next order, we find $\mu_{1 / 2}$ is equal to the constant $\bar{\mu}$, and

$$
-\left(\begin{array}{c}
\Phi_{1 / 2} \\
\Psi_{1 / 2}
\end{array}\right)_{\zeta \zeta}+\nabla^{2} W\left(\Phi_{0}, \Psi_{0}\right)\left(\begin{array}{c}
\Phi_{1 / 2} \\
\Psi_{1 / 2}
\end{array}\right)=\left(\begin{array}{c}
\bar{\mu} \\
\nu_{\infty}
\end{array}\right) .
$$

Solvability of this equation yields the relationship $(1-f) \bar{\mu}+$ $f v_{\infty}=0$. Matching to region III determines the constants in (A28) and (A29) as

$$
c_{-}=-\gamma v_{\infty} /\left\{\begin{array}{ll}
I_{0}(r / \sqrt{\beta}), & d=2 \\
\sinh (r / \sqrt{\beta}) / r, & d=3
\end{array},\right.
$$

and

$$
c_{+}=-\gamma v_{\infty} /\left\{\begin{array}{ll}
K_{0}(r / \sqrt{\beta}), & d=2 \\
\exp (-r / \sqrt{\beta}) / r, & d=3
\end{array},\right.
$$

where $\gamma$ is defined in (11). Further expansion gives $\left(\mu_{1}\right)_{\zeta \zeta}=$ 0 and $\left(\mu_{3 / 2}\right)_{\zeta \zeta}=0$. Integration and matching the former to regions $\mathrm{III}_{ \pm}$implies $\mu_{1}(\zeta)$ is a constant, whereas integrating and matching the later implies continuity of the derivative

$$
\mu_{1 \rho}(\rho= \pm w)=\left(\mu_{1 / 2}\right)_{r}(r= \pm R)
$$

The leading order solution in region $\mathrm{I}$ is the same as before. The next order solves $\left(\mu_{1 / 2}\right)_{\rho \rho}=0$ and $\left(\nu_{1 / 2}\right)_{\rho \rho}=0$, which by matching to regions $\mathrm{II}_{ \pm}$imply that $\mu_{1 / 2}=\bar{\mu}$ and $v_{1 / 2}=$ $v_{\infty}$. The next order problem is $\left(v_{1}\right)_{\rho \rho}=1-f$, whose general solution is

$$
\mu_{1}=\frac{(1-f) \rho^{2}}{2}+A \rho+B
$$

Combining this with (A33), (A28), and (A29),

$$
(1-f) w+A=\frac{c_{+}}{\beta} \begin{cases}K_{0}^{\prime}(R / \sqrt{\beta}), & d=2 \\ \exp (-R / \sqrt{\beta})\left(-1 / R-\sqrt{\beta} / R^{2}\right), & d=3\end{cases}
$$

and

$$
-(1-f) w+A=\frac{c_{-}}{\beta}\left\{\begin{array}{ll}
I_{0}^{\prime}(R / \sqrt{\beta}), & d=2 \\
\cosh (R / \sqrt{\beta}) / R-\sqrt{\beta} \sinh (R / \sqrt{\beta}) / R^{2}, & d=3
\end{array} .\right.
$$

These equations determine $w$ and $A$ as functions of $R$,

$$
w=v_{\infty} \bar{w}, \quad \bar{w}(R)=\frac{\gamma}{2(1-f) \sqrt{\beta}} \begin{cases}I_{0}^{\prime}(R / \sqrt{\beta}) / I_{0}(R / \sqrt{\beta})-K_{0}^{\prime}(R / \sqrt{\beta}) / K_{0}(R / \sqrt{\beta}), & d=2 \\ 1+\operatorname{coth}(R / \sqrt{\beta}), & d=3\end{cases}
$$

and

$$
A=v_{\infty} \bar{A}, \quad \bar{A}(R)=-\frac{\gamma}{2 \sqrt{\beta}}\left\{\begin{array}{ll}
I_{0}^{\prime}(R / \sqrt{\beta}) / I_{0}(R / \sqrt{\beta})+K_{0}^{\prime}(R / \sqrt{\beta}) / K_{0}(R / \sqrt{\beta}), & d=2 \\
-1-2 \sqrt{\beta} / R+\operatorname{coth}(R / \sqrt{\beta}), & d=3
\end{array} .\right.
$$

Continuing the expansion in region II,

$$
\mathcal{L}\left(\begin{array}{c}
\Phi_{1} \\
\Psi_{1}
\end{array}\right)=\left(\begin{array}{c}
\mu_{1} \\
0
\end{array}\right)+\frac{d-1}{R}\left(\begin{array}{c}
\Phi_{0} \\
\Psi_{0}
\end{array}\right)_{\zeta}+\boldsymbol{q}, \quad \boldsymbol{q} \equiv-\frac{1}{2} \nabla^{3} W\left(\Phi_{0}, \Psi_{0}\right) \cdot\left(\begin{array}{c}
\Phi_{1 / 2} \\
\Psi_{1 / 2}
\end{array}\right) \cdot\left(\begin{array}{c}
\Phi_{1 / 2} \\
\Psi_{1 / 2}
\end{array}\right) .
$$

Solvability of this equation is

$$
\mu_{1}(1-f)= \pm \frac{\sigma(d-1)}{R}+Q, \quad Q \equiv \int_{-\infty} \boldsymbol{q} \cdot\left(\begin{array}{l}
\Phi_{h} \\
\Psi_{h}
\end{array}\right)_{\zeta} d \zeta
$$

where + is chosen for region $\mathrm{II}_{+}$and - for $\mathrm{II}_{-}$. Returning to the solution for $\mu_{1}(\rho)$ in (A34), matching to both regions $\mathrm{II}_{ \pm}$ using (A40) leads to (14).

\section{APPENDIX B: DERIVATION OF THE DYNAMIC FREE BOUNDARY PROBLEM}

For an arbitrary configuration, the interface region (still denoted region II) is described using a a scaled, moving, fitted coordinate system $(\zeta, s)$, defined so that $\zeta$ is the signed distance, measured in units of $\epsilon$, from the interface, and $s$ is a transverse coordinate (or coordinates). The orientation is chosen so that $\zeta \rightarrow-\infty$ as the region between interfaces is approached. Region I, described using the scaled coordinates $(X, Y, Z)$ now includes both the domain between interfaces and an $O\left(\epsilon^{1 / 3}\right)$ region outside. The normal velocity $v_{n}$ measures the motion of the interface (with respect to original length scale) on a slow timescale $\tau=t \epsilon^{1 / 3}$. The scaling of $\nu_{\infty}$ 
is prescribed in advance to be $O\left(\epsilon^{1 / 3}\right)$, which as will be shown leads to a balance of polymer and surface tension effects. All quantities are expanded in powers of $\epsilon^{1 / 3}$. The other details are geometry dependent and explained in the following sections.

\section{Bilayer configurations}

The outermost layer, region III, has a leading order solution which is the same as the equilibrium problem. In terms of the variable $\mu$ this reads

$$
\left(\mu_{1 / 3}\right)_{x x}=\left(\mu-\mu_{\infty}\right) / \beta,
$$

whose solution is

$$
\mu_{1 / 3}=C \exp (-|x| / \sqrt{\beta})+\mu_{\infty},
$$

valid for $|x| \gg \epsilon^{1 / 3}$.

In region II, the solutions at the first two orders are the same as in Appendix A 1, with the exception that $\Phi_{1 / 3}(\zeta)$ and $\Psi_{1 / 3}(\zeta)$ are bounded, and therefore the solvability condition is simply

$$
\left(\begin{array}{c}
\bar{\mu} \\
v_{\infty}
\end{array}\right) \cdot\left(\begin{array}{c}
1-f \\
f
\end{array}\right)=0 .
$$

Matching $\mu$ to region III gives

$$
C=-f v_{\infty} /(1-f)-\mu_{\infty} .
$$

At $O\left(\epsilon^{2 / 3}\right)$ one finds that $\mu_{2 / 3}(\zeta)$ and $\nu_{2 / 3}(\zeta)$ are constants, and

$$
\begin{aligned}
\mathcal{L}\left(\begin{array}{l}
\Phi_{1} \\
\Psi_{1}
\end{array}\right)= & \left(\begin{array}{c}
\mu_{2 / 3} \\
\nu_{2 / 3}
\end{array}\right) \mp \kappa\left(\begin{array}{l}
\Phi_{0} \\
\Psi_{0}
\end{array}\right)_{\zeta}-\nabla^{3} W\left(\Phi_{h}, \Psi_{h}\right) \\
& \times\left(\begin{array}{l}
\Phi_{1 / 3} \\
\Psi_{1 / 3}
\end{array}\right) \cdot\left(\begin{array}{l}
\Phi_{1 / 3} \\
\Psi_{1 / 3}
\end{array}\right) .
\end{aligned}
$$

The interface curvature is defined here so that $\kappa<0$ when the region between interfaces is convex. Solvability gives

$$
\left(\begin{array}{l}
\mu_{2 / 3} \\
\nu_{2 / 3}
\end{array}\right) \cdot\left(\begin{array}{c}
1-f \\
f
\end{array}\right)=-\sigma \kappa+\delta,
$$

where $\delta$ is an ultimately irrelevant constant accounting for the $\nabla^{3} W$ term.

In region $\mathrm{I}$, the $O\left(\epsilon^{2 / 3}\right)$ problems are

$$
\Delta v_{2 / 3}=\Phi_{0}, \quad \Delta v=0, \quad \Delta \equiv \partial_{X X}+\partial_{Y Y}+\partial_{Z Z},
$$

where $\Phi_{0}=1-f$ between the interfaces and $\Phi_{0}=0$ otherwise. Matching to region II provides a boundary condition for (B7), namely, (B6). In addition, matching to region III yields

$$
\mu_{2 / 3 X} \sim \mp C / \sqrt{\beta}, \quad X \rightarrow \pm \infty .
$$

Dynamics are obtained from the next expansion term in region II,

$$
-v_{n}\left(\begin{array}{l}
\Phi_{0} \\
\Psi_{0}
\end{array}\right)_{\zeta}=\left(\begin{array}{c}
\mu_{4 / 3} \\
v_{4 / 3}
\end{array}\right)_{\zeta \zeta},
$$

whose solvability provides the condition

$$
v_{n}\left(\begin{array}{c}
1-f \\
f
\end{array}\right)=\left.\left(\begin{array}{c}
\mu_{4 / 3 \zeta} \\
v_{4 / 3 \zeta}
\end{array}\right)\right|_{-\infty} ^{\infty} .
$$

Matching to region I gives

$$
v_{n}\left(\begin{array}{c}
1-f \\
f
\end{array}\right)=\left[\frac{\partial}{\partial n}\left(\begin{array}{l}
\mu_{2 / 3} \\
v_{2 / 3}
\end{array}\right)\right],
$$

where [] is the difference of a quantity from the outside region to the region between interfaces.

The time-independent solution has interfaces at $X= \pm w_{0}$, and

$$
\mu_{2 / 3 X}^{0}=\left\{\begin{array}{ll}
(1-f) X, & |X|<w_{0} \\
\mp C / \sqrt{\beta}, & \pm X>w
\end{array} .\right.
$$

In addition, $\mu_{2 / 3 X}$ is continuous across interfaces, so that using (B4),

$$
\bar{v}=\frac{1-f}{f}\left[(1-f) w_{0} \sqrt{\beta}+\mu_{\infty}\right],
$$

which is consistent with the more general result (11) for $w_{0}=$ $O\left(\epsilon^{1 / 3}\right)$.

The conditions (B6)-(B9), and (B11) represent a dynamic free boundary problem for the two interfaces constituting a bilayer. This may be simplified by the introduction of the variable $V \equiv(1-f) \mu_{2 / 3}+f \nu_{2 / 3}-\delta$, and a rescaled time $\tau \rightarrow \tau\left((1-f)^{2}+f^{2}\right)$, giving the stated problem (17)-(19) together with the far-field condition

$$
\lim _{X \rightarrow \pm \infty} V_{X}=\mp C(1-f) / \sqrt{\beta},
$$

where the constant $C$ given by (B4).

\section{Cylinders}

The dynamic free boundary problem is derived using the same expansion as for the equilibrium problem (Appendix A 2); however, the scale for $R(\epsilon)$ is prescribed in advance to be $\epsilon^{1 / 3}$. This sets the scale for cylindrical variables $\rho=r / \epsilon^{1 / 3}$ and $Y=y / \epsilon^{1 / 3}$. The expansion at order $*$ is therefore $O[\epsilon / R(\epsilon)]=O\left[R(\epsilon)^{2} \ln R(\epsilon)\right]$, and at order $* *$ is $O\left[R(\epsilon)^{2}\right]$.

The solutions in region III are the same as in equilibrium, with $\mu_{*}=\mu_{\infty}, v_{*}=v_{\infty}, v_{* *}=0$ and $v_{* *}=C K_{0}(r / \sqrt{\beta})$. The expansion terms in region II yield solvability conditions

$$
\left(\begin{array}{c}
\mu_{*} \\
\nu_{*}
\end{array}\right) \cdot\left(\begin{array}{c}
1-f \\
f
\end{array}\right)=-\sigma \kappa
$$

and

$$
\left(\begin{array}{c}
\mu_{* *} \\
v_{* *}
\end{array}\right) \cdot\left(\begin{array}{c}
1-f \\
f
\end{array}\right)=0 .
$$

Here $\kappa$ is the sum of principle interface curvatures. At order $\epsilon R(\epsilon)$ in region II, an expression for the normal interface velocity equivalent to (B9) arises. The region I expansion also occurs on two levels, giving $\Delta \mu_{*}=0=\Delta \nu_{*}, \Delta \nu_{* *}=0$, and $\Delta v_{* *}=\Phi_{0}$, with $\Delta$ being the Laplacian in scaled coordinates.

Matching from regions III to I produces

$$
\begin{aligned}
\mu_{*}(\rho) & \sim \mu_{\infty}-C, \quad \mu_{* *}(\rho) \sim-C \ln \rho+C \ln (2 \sqrt{\beta}), \\
\rho & \rightarrow \infty,
\end{aligned}
$$

as well as $\nu_{*}(\rho) \sim v_{\infty}$ and $\nu_{* *} \sim 0$ as $\rho \rightarrow \infty$. Note this implies that $\rho \mu_{\rho} \sim-C$, which may be used to eliminate $C$ from these conditions. 
As above, the free boundary problems may be reformulated into a single one by setting Setting $V=(1-f)\left(\mu_{*}+\mu_{* *}\right)+$ $f\left(\mu_{*}+\mu_{* *}\right)$. The result is the same as (17)-(19), and now the far-field condition can be written

$$
\lim _{\rho \rightarrow \infty}\left(V-\rho V_{\rho} \ln \rho\right)=\ln (2 \sqrt{\beta}) \rho V_{\rho}+f v_{\infty} .
$$

\section{Spheres}

The derivation of a free boundary problem for roughly spherical interfaces closely follows the previous sections, and the only unique aspect is the far-field condition. Matching from region I to region III results in

$$
\mu_{2 / 3}(\rho) \sim \mu_{\infty}, \quad \mu_{2 / 3}(\rho) \sim v_{\infty}, \quad \rho \rightarrow \infty
$$

in contrast to (B8) in the bilayer case. Defining $V=(1-$ f) $\mu_{2 / 3}+f v_{2 / 3}$ as before leads to (17)-(19) together with

$$
\lim _{\rho \rightarrow \infty} V=(1-f) \mu_{\infty}+f v_{\infty}
$$

[1] S. J. Holder and N. A. J. M. Sommerdijk, New micellar morphologies from amphiphilic block copolymers: Disks, toroids and bicontinuous micelles, Polym. Chem. 2, 1018 (2011).

[2] A. Blanazs, S. P. Armes, and A. J. Ryan, Self-assembled block copolymer aggregates: From micelles to vesicles and their biological applications, Macromol. Rapid Commun. 30, 267 (2009).

[3] Y. Mai and A. Eisenberg, Self-assembly of block copolymers, Chem. Soc. Rev. 41, 5969 (2012).

[4] V. Malinova, S. Belegrinou, D. de Bruyn Ouboter, and W. P. Meier, Biomimetic block copolymer membranes, in Organic Electronics (Springer, Berlin, Heidleberg, 2010), pp. 213-258.

[5] J. Yuan and A. H. E. Müller, One-dimensional organic-inorganic hybrid nanomaterials, Polymer 51, 4015 (2010).

[6] J. G. E. M. Fraaije and G. J. A. Sevink, Model for pattern formation in polymer surfactant nanodroplets, Macromolecules 36, 7891 (2003).

[7] D. E. Discher and A. Eisenberg, Polymer vesicles, Science 297, 967 (2002).

[8] S. Jain and F. S. Bates, On the origins of morphological complexity in block copolymer surfactants, Science 300, 460 (2003).

[9] S. Jain and F. S. Bates, Consequences of nonergodicity in aqueous binary PEO-PB micellar dispersions, Macromolecules 37, 1511 (2004).

[10] R. G. Larson, Monte Carlo lattice simulation of amphiphilic systems in two and three dimensions, J. Chem. Phys. 89, 1642 (1988).

[11] H. Noguchi and M. Takasu, Self-assembly of amphiphiles into vesicles: A Brownian dynamics simulation, Phys. Rev. E 64, 041913 (2001).

[12] S. Yamamoto, Y. Maruyama, and S.-A. Hyodo, Dissipative particle dynamics study of spontaneous vesicle formation of amphiphilic molecules, J. Chem. Phys. 116, 5842 (2002).

[13] G. Gompper and M. Schick, Correlation between Structural and Interfacial Properties of Amphiphilic Systems, Phys. Rev. Lett. 65, 1116 (1990).

[14] K. Promislow and L. Yang, Existence of compressible bilayers in the functionalized Cahn-Hilliard equation, SIAM J. Appl. Dyn. Syst. 13, 629 (2014).

[15] M. W. Matsen and F. S. Bates, Unifying weak- and strongsegregation block copolymer theories, Macromolecules 29, 1091 (1996).

[16] F. S. Bates and G. H. Fredrickson, Block copolymersDesigner soft materials, Phys. Today 52, 32 (1999).
[17] G. H. Fredrickson, The Equilibrium Theory of Inhomogeneous Polymers, Oxford Science Publications (Clarendon Press, Oxford, 2006).

[18] E. Helfand, Theory of inhomogeneous polymers: Fundamentals of the Gaussian random-walk model, J. Chem. Phys. 62, 999 (1975).

[19] J. G. E. M. Fraaije, Dynamic density functional theory for microphase separation kinetics of block copolymer melts, J. Chem. Phys. 99, 9202 (1993).

[20] N. M. Maurits and J. G. E. M. Fraaije, Mesoscopic dynamics of copolymer melts: From density dynamics to external potential dynamics using nonlocal kinetic coupling, J. Chem. Phys. 107, 5879 (1997).

[21] X. He and F. Schmid, Dynamics of spontaneous vesicle formation in dilute solutions of amphiphilic diblock copolymers, Macromolecules 39, 2654 (2006).

[22] X. He and F. Schmid, Spontaneous Formation of Complex Micelles from a Homogeneous Solution, Phys. Rev. Lett. 100, 137802 (2008).

[23] L. Leibler, Theory of microphase separation in block copolymers, Macromolecules 13, 1602 (1980).

[24] T. Ohta and K. Kawasaki, Equilibrium morphology of block copolymer melts, Macromolecules 19, 2621 (1986).

[25] R. Choksi and X. Ren, On the derivation of a density functional theory for microphase separation of diblock copolymers, J. Statist. Phys. 113, 151 (2003).

[26] T. Uneyama and M. Doi, Density functional theory for block copolymer melts and blends, Macromolecules 38, 196 (2005).

[27] Y. Nishiura and I. Ohnishi, Some mathematical aspects of the micro-phase separation of diblock copolymers, Physica D 84, 31 (1995).

[28] M. Müller and J. C. Orozco Rey, Continuum models for directed self-assembly, Mol. Syst. Des. Eng. 3, 295 (2018).

[29] R. Choksi, M. A. Peletier, and J. F. Williams, On the phase diagram for microphase separation of diblock copolymers: An approach via a nonlocal Cahn-Hilliard functional, SIAM J. Appl. Math. 69, 1712 (2009).

[30] E. Avalos, T. Higuchi, T. Teramoto, H. Yabu, and Y. Nishiura, Frustrated phases under three-dimensional confinement simulated by a set of coupled Cahn-Hilliard equations, Soft Matter 12, 5905 (2016).

[31] K. Glasner, Evolution and competition of block copolymer nanoparticles, SIAM J. Appl. Math. 79, 28 (2019).

[32] T. Uneyama and M. Doi, Calculation of the micellar structure of polymer surfactant on the basis of the density functional theory, Macromolecules 38, 5817 (2005). 
[33] T. Uneyama, Density functional simulation of spontaneous formation of vesicle in block copolymer solutions, J. Chem. Phys. 126, 114902 (2007).

[34] J. W. Cahn and J. E. Hilliard, Free energy of a nonuniform system I. Interfacial free energy, J. Chem. Phys. 28, 258 (1957).

[35] I. M. Lifshitz and V. V. Slyozov, The kinetics of precipitation from supersaturated solid solutions, J. Chem. Phys. Solids 19, 35 (1961).

[36] C. Wagner, Theorie for Alterung von Niederschlagen durch Umlosen, Z. Elektrochem. 65, 581 (1961).

[37] T. Ohta and M. Nonomura, Elastic property of bilayer membrane in copolymer-homopolymer mixtures, Eur. Phys. J. B 2, 57 (1998).

[38] K. Glasner, Multilayered equilibria in a density functional model of copolymer-solvent mixtures, SIAM J. Math. Anal. 49, 1593 (2017).

[39] R. Choksi and X. Ren, Diblock copolymer/homopolymer blends: Derivation of a density functional theory, Phys. D 203, 100 (2005).

[40] R. L. Pego, Front migration in the nonlinear Cahn-Hilliard equation, Proc. R. Soc. Lond. A 422, 261 (1989).

[41] P. Carter and K. Glasner, Existence and stability of amphiphilic equilibria in a density functional model (unpublished).

[42] K. Glasner and S. Orizaga, Improving the accuracy of convexity splitting methods for gradient flow equations, J. Comput. Phys. 315, 52 (2016).

[43] U. Seifert, Configurations of fluid membranes and vesicles, Adv. Phys. 46, 13 (1997).

[44] T. F. Zhu, K. Adamala, N. Zhang, and J. W. Szostak, Photochemically driven redox chemistry induces protocell membrane pearling and division, Proc. Natl. Acad. Sci. USA 109, 9828 (2012).

[45] A. N. Semenov, Theory of block copolymer interfaces in the strong-segregation limit, Macromolecules 26, 6617 (1993).

[46] Y. Yu, L. Zhang, and A. Eisenberg, Multiple morphologies of crew-cut aggregates of polybutadiene- $b$-poly (acrylic acid) diblocks with low $T_{g}$ cores, Langmuir 13, 2578 (1997).

[47] Y.-Y. Won, A. K. Brannan, H. T. Davis, and F. S. Bates, Cryogenic transmission electron microscopy (cryo-tem) of micelles and vesicles formed in water by poly (ethylene oxide)-based block copolymers, J. Phys. Chem. B 106, 3354 (2002).

[48] A. Christlieb, N. Kraitzman, and K. Promislow, Competition and complexity in amphiphilic polymer morphology, Physica D: Nonlinear Phenomenon 400, 132144 (2019).

[49] P. B. Canham, The minimum energy of bending as a possible explanation of the biconcave shape of the human red blood cell, J. Theor. Biol. 26, 61 (1970).

[50] W. Helfrich, Elastic properties of lipid bilayers: Theory and possible experiments, Z. Naturforsch. C 28, 693 (1973).

[51] Lord Rayleigh, On the instability of jets, Proc. Lond. Math. Soc. S1, 4 (1878).

[52] S. Mora, T. Phou, J.-M. Fromental, L. M. Pismen, and Y. Pomeau, Capillarity Driven Instability of a Soft Solid, Phys. Rev. Lett. 105, 214301 (2010).

[53] C. Xuan and J. Biggins, Plateau-Rayleigh instability in solids is a simple phase separation, Phys. Rev. E 95, 053106 (2017).

[54] A. Doelman, G. Hayrapetyan, K. Promislow, and B. Wetton, Meander and pearling of single-curvature bilayer interfaces in the functionalized Cahn-Hilliard equation, SIAM J. Math. Anal. 46, 3640 (2014).

[55] P. E. Theodorakis, W. Paul, and K. Binder, Pearl-necklace structures of molecular brushes with rigid backbone under poor solvent conditions: A simulation study, J. Chem. Phys. 133, 104901 (2010).

[56] R. Bar-Ziv and E. Moses, Instability and "Pearling" States Produced in Tubular Membranes by Competition of Curvature and Tension, Phys. Rev. Lett. 73, 1392 (1994).

[57] Q. Du, C. Liu, and X. Wang, A phase field approach in the numerical study of the elastic bending energy for vesicle membranes, J. Comput. Phys. 198, 450 (2004).

[58] M. A. Peletier and M. Röger, Partial localization, lipid bilayers, and the elastica functional, Arch. Rational Mech. Anal. 193, 475 (2009).

[59] K. B. Glasner and A. E. Lindsay, The stability and evolution of curved domains arising from one-dimensional localized patterns, SIAM J. Appl. Dyn. Syst. 12, 650 (2013).

[60] S. Baldo, Minimal interface criterion for phase transitions in mixtures of Cahn-Hilliard fluids, in Annales de l'IHP Analyse non linéaire (Elsevier Masson, 1990), Vol. 7, pp. 67-90. 